\title{
Strategic experimentation with Poisson bandits
}

\author{
Godfrey Keller \\ Department of Economics, University of Oxford \\ Sven Rady \\ Department of Economics, University of Munich
}

\begin{abstract}
We study a game of strategic experimentation with two-armed bandits where the risky arm distributes lump-sum payoffs according to a Poisson process. Its intensity is either high or low, and unknown to the players. We consider Markov perfect equilibria with beliefs as the state variable and show that all such equilibria exhibit an "encouragement effect" relative to the single-agent optimum. There is no equilibrium in which all players use cutoff strategies. Owing to the encouragement effect, asymmetric equilibria in which players take turns playing the risky arm before all experimentation stops Pareto dominate the unique symmetric equilibrium. Rewarding the last experimenter with a higher continuation value increases the range of beliefs where players experiment, but may reduce the intensity of experimentation at more optimistic beliefs. This suggests that there is no equilibrium that uniformly maximizes the players' average payoff.
\end{abstract}

Keywords. Strategic experimentation, two-armed bandit, Poisson process, Bayesian learning, piecewise deterministic process, Markov perfect equilibrium, differential-difference equation.

JEL CLASSIFICATION. C73, D83, O32.

\section{INTRODUCTION}

When firms cooperate in a research joint venture, each faces a dynamic problem in which it can perform repeated costly experiments (that is, spend time, effort, and money on the purported innovation) but also learn from the experimental observations of the

Godfrey Keller: godfrey .keller@economics.ox.ac.uk

Sven Rady: sven.rady@lrz.uni-muenchen.de

Our thanks for very helpful comments and suggestions are owed to four anonymous referees, Martin Cripps, Johannes Hörner, Jörg Oechssler, and to seminar participants at the universities of Mainz, Mannheim, Munich, Nürnberg, Oxford, Toulouse, Wisconsin-Madison, ETH Zurich, the 2007 Annual Meeting of the Society for Economic Dynamics in Prague, the SFB/TR 15 Conference on Strategic Information Acquisition and Transmission (Munich, November 2008), and the 2009 North American Summer Meeting of the Econometric Society in Boston. We thank the Institut d'Economie Industrielle at the University of Toulouse, the Center for Economic Studies at the University of Munich, the Business and Public Policy Group at the Wharton School, and the Social Science Research Center Berlin (WZB) for their hospitality. Financial support from the Deutsche Forschungsgemeinschaft through SFB/TR 15 and GRK 801 and from the Bavarian Graduate Program in Economics is gratefully acknowledged.

Copyright () 2010 Godfrey Keller and Sven Rady. Licensed under the Creative Commons AttributionNonCommercial License 3.0. Available at http: //econtheory . org.

DOI: $10.3982 /$ TE595 
others. Such a game of strategic experimentation arises in a variety of economic contexts; besides firms' research and development (R\&D) activities, consumer search or experimental consumption of a new product are prominent examples. Academic researchers pursuing a common research agenda or simply working on a joint paper are also effectively engaged in strategic experimentation.

In this paper we analyze a game of strategic experimentation where a finite number of players face identical two-armed bandit problems. There is a safe arm that offers a known and constant flow payoff and a risky arm whose lump-sum payoffs are driven by a Poisson process of unknown intensity. The risky arm can be either good or bad: if it is good, the lump sums arrive more frequently than if it is bad. While all risky arms are of the same type (all good or all bad), lump sums arrive independently across players. Each player is endowed with a stream of one unit of a perfectly divisible resource and, at each point in time, must decide how to split this resource between the two arms. Players' actions and outcomes are publicly observed, so there are perfect informational spillovers between players.

With Poisson bandits, news arrives in a "lumpy" fashion. Examples are the occasional breakthrough in research and development, a completed research paper in a longer-term research agenda, or one of a sequence of crucial proofs in a paper. Beliefs jump to a more optimistic level whenever a "news event" or "success" occurs, whereas they gradually become more pessimistic in between such events.

A single success on the risky arm does not fully reveal its type. This stands in marked contrast to the experimentation model of Keller et al. (2005). There, a good risky arm also generates lump-sum payoffs according to some Poisson process, but a bad risky arm never generates any payoffs, so the belief jumps all the way to certainty as soon as the first lump sum arrives, irrespective of the belief held immediately before. ${ }^{1}$ In the present model, there is never certainty about the state of the risky arm, and the belief held immediately after a success varies with the belief held immediately before. As a consequence, when the players in our model use Markov strategies with the posterior belief as the state variable, their payoff functions solve first-order differential-difference equations. Despite this technical complication, these equations can be analyzed by elementary methods and admit closed-form solutions.

While all Markov perfect equilibria (MPE) of the experimentation game are inefficient because of free-riding, our first main contribution is to show that they always exhibit an encouragement effect: the presence of other players encourages at least one of them to continue experimenting with the risky arm at beliefs where a single agent would already have given up. This effect rests on two conditions: the experimentation by any pioneer contemplating the use of the risky arm beyond the single-agent optimum must increase the likelihood that other players will return to the risky arm in the future, and these future actions must be valuable to the pioneer. The encouragement effect was first described by Bolton and Harris (1999) in a model where the risky arm yields a flow payoff with Brownian noise. Focusing on the symmetric MPE of their model, however, they

\footnotetext{
${ }^{1}$ For any time-invariant positive allocation of resources to the risky arms, this resolution of uncertainty happens at an exponentially distributed random time. Following Keller et al. (2005), we therefore refer to that model as the exponential model and to the present model as the Poisson model.
} 
obtain the encouragement effect for this particular equilibrium only. In contrast, we are able to establish this effect for all Markov perfect equilibria of the Poisson model.

We then show that there is no MPE in which all players use cutoff strategies, i.e., use the risky arm exclusively when the probability they assign to the risky arm being good is above some cutoff, and use the safe arm when it is below. In fact, the player who is supposed to use the least optimistic cutoff in a purported MPE in cutoff strategies always has an incentive to deviate to the safe action at the second least optimistic cutoff, where one of the other players is supposed to switch action.

A symmetric MPE thus necessarily requires the players to choose an interior allocation of their resource at some beliefs. We show that the Poisson model admits a unique symmetric MPE. As in its counterpart in the Bolton-Harris model, all players use the risky arm exclusively when they are sufficiently optimistic, the safe arm when they are sufficiently pessimistic, and both arms simultaneously at intermediate beliefs. Further, the acquisition of information is slowed down so severely near the lower bound of the intermediate range that the players' beliefs cannot reach this bound in finite time.

This strongly suggests that asymmetric equilibria where a last experimenter keeps the rate of information acquisition bounded away from zero at pessimistic beliefs ought to be more efficient than symmetric equilibrium. Bolton and Harris (2000), who study the undiscounted limit of the Brownian model, and Keller et al. (2005) confirm this by constructing a variety of asymmetric MPE that dominate the symmetric MPE in terms of aggregate payoffs. However, they do so in environments without the encouragement effect. In both cases, it is the second of the two conditions stated above that fails. In the undiscounted Brownian model, the catching-up criterion implies that best responses do not depend on players' continuation values, so there is no channel through which the opponents' future actions can influence a player's current optimal choice. In the exponential model, the only way for the pioneer to make the other players return to the risky arm is to have a success himself, but since such a success is fully revealing, he will then not benefit at all from his opponents' future actions.

The construction of asymmetric equilibria in Keller et al. (2005) relies on a backwardinduction approach anchored at the single-agent cutoff belief. The presence of the encouragement effect obviously rules out such an approach in the Poisson model. The construction of asymmetric equilibria is further complicated by the "nonlocal" nature of the problem: each player's payoff depends not only on the action profiles and continuation values prevailing in a neighborhood of the current belief, but also on the continuation value at the belief that would be reached after a success on a risky arm. Our second main contribution is to provide a constructive solution to this problem.

The approach that we use here rests on two ideas. The first is to give the players a common continuation value after any success on a risky arm; the second is to let them alternate finitely many times between the roles of experimenter and free-rider before all experimentation stops. Assigning common continuation values after successes allows us to construct the players' average payoff function before assigning individual strategies, which simplifies the problem considerably. Letting players take turns playing risky 
allows us to achieve an overall intensity of experimentation higher than in the symmetric equilibrium, hence higher average equilibrium payoffs. We show that this alternation can actually be performed in a way that leads to a Pareto improvement over the symmetric equilibrium.

In the exponential model, finite switching between the safe and the risky arms is also able to generate higher average equilibrium payoffs than the symmetric MPE, but not a Pareto improvement over this equilibrium. The reason is that in the absence of the encouragement effect, the last experimenter quits at the same belief (the single-agent cutoff) at which all players stop experimenting in the symmetric equilibrium; bearing all the costs of experimentation on his own, the last experimenter is then necessarily worse off than under symmetry immediately to the right of this cutoff. Because of the encouragement effect in the Poisson model, by contrast, sufficiently frequent but finite switching between the roles of experimenter and free-rider brings every player's payoff function close enough to the average payoff function so that even the last experimenter is better off than in the symmetric MPE. ${ }^{2}$

Ideally, we would like to have a complete characterization at any given belief of the set of payoff profiles and average payoffs that can be achieved in a Markov perfect equilibrium of the experimentation game. In the exponential model with finite switching, such a characterization is available at least in the two-player case. Our third main contribution is to explore whether a similar characterization can be obtained in the Poisson model.

With two players, the exponential model actually admits uniformly best and worst equilibria that achieve the maximal (resp. minimal) intensity of experimentation and the maximal (resp. minimal) average payoff compatible with Markov perfection and finite switching, and do so on the entire unit interval. While the worst MPE is the symmetric one, the best equilibria have the same structure as the asymmetric MPE that we construct in the Poisson model; all these equilibria are thus easily seen to be the limits of two-player equilibria of the Poisson model for vanishing arrival rates of lump-sum payoffs on a bad risky arm.

Moreover, there is a "most inequitable" two-player MPE in the exponential model such that the payoffs achieved by the last experimenter and the last free-rider constitute a uniform lower and upper bound, respectively, on the individual equilibrium payoffs that are compatible with Markov perfection and finite switching. These equilibria involve what we call simple strategies, that is, strategies that have each player use one or the other arm exclusively at any given belief. To investigate whether these most inequitable equilibria can also be obtained as limits of two-player equilibria of the Poisson model, we first construct a benchmark simple MPE where the players obtain common continuation payoffs after any success on a risky arm. In a numerical example, we then progressively diverge from this benchmark by rewarding the last experimenter, that is,

\footnotetext{
${ }^{2}$ To obtain a Pareto improvement over the symmetric MPE in the exponential model, one has to construct an equilibrium without a last experimenter, that is, one where players switch actions infinitely often over a finite time interval; cf. Section 6.2 of Keller et al. (2005). As infinite switching is not required for such a Pareto improvement in the Poisson model, we do not consider it here and instead restrict players to Markov strategies with finitely many discontinuities.
} 
by giving him a higher continuation value after a success than his opponent. This yields equilibria with progressively increasing payoff asymmetry between the players and in the limit replicates the most inequitable equilibria of the exponential model.

We find that rewarding the last experimenter raises average payoffs at relatively pessimistic beliefs (because the last experimenter is willing to play risky over a larger range of beliefs), but lowers them at optimistic beliefs (because the intensity of experimentation drops from 2 to 1 earlier when the difference between the players' equilibrium payoffs increases). This means, in particular, that although we can approach the best twoplayer equilibria of the exponential model arbitrarily closely with asymmetric equilibria of the Poisson model, the latter do not constitute uniformly best equilibria themselves. More generally, to the extent that we can always reward the last experimenter somewhat more, this suggests the conjecture that the Poisson model admits no uniformly best MPE. ${ }^{3}$

In the alternation phase of some of the examples we calculate, we observe an anticipation effect that is already present in the infinite-switching equilibria of Keller et al. (2005): the value function of a lone experimenter decreases in the current belief over some range. The intuition for this effect is that, conditional on no impending success on his own risky arm, the player will soon be able to enjoy a free ride, and the lower the current belief, the sooner this time will come.

For each type of equilibrium that we construct, we provide representations of the players' payoff functions that are explicit up to some constant of integration. While these representations cannot be used to derive comparative statics results, say, they greatly simplify the computation of numerical examples. Asymmetric equilibria with common continuation values after a success just require a one-dimensional search for an implicitly defined constant of integration. In the two-player equilibria that reward the last experimenter, we have to solve for two of these constants. Either task is easily carried out in a spreadsheet.

The Poisson model is a natural analog in continuous time of the two-outcome bandit model in Rothschild (1974), the first paper to use the bandit framework in economics; see Bergemann and Välimäki (2008) for a survey of the ensuing literature. Through its focus on bandit learning as a canonical model of strategic experimentation in teams, our paper is most closely related to Bolton and Harris $(1999,2000)$ and Keller et al. (2005), sharing with them the assumption that the players face risky arms of a common type. Klein and Rady (2008) and Klein (2010), by contrast, consider two players who face risky arms of opposite types, one good and one bad. Strulovici (2010) studies majority voting in a collective decision problem where the type of the risky arm varies across individuals.

Our paper is further related to a strand of the industrial organization literature that studies R\&D investment games under learning. Malueg and Tsutsui (1997) investigate a model of a patent race with learning where the arrival time of an innovation is exponentially distributed given the stock of knowledge, implying the same deterministic belief

\footnotetext{
${ }^{3}$ This does not preclude the possibility that for each belief, there exists a Markov perfect equilibrium that maximizes average payoffs when the game is started at this particular belief. An investigation of this possibility and of the upper envelope of average equilibrium payoffs is beyond the scope of this paper.
} 
revision prior to the innovation as our model exhibits in between lump sums. Building on the exponential bandit framework of Keller et al. (2005), Besanko and Wu (2008) study the effects of post-innovation market structure on cooperative and competitive R\&D investments, respectively. Décamps and Mariotti (2004), Hopenhayn and Squintani (2008), and Moscarini and Squintani (2007) all analyze models where news arrives in the form of the increments of a (compound) Poisson process; as they consider stopping games with private information, however, the resulting strategic interactions are very different from that in our model.

The remainder of the paper is organized as follows. Section 2 sets up the Poisson bandit model. Section 3 establishes the efficient benchmark. Section 4 introduces the strategic problem, establishes the encouragement effect, and proves the impossibility of cutoff equilibria. Section 5 presents the unique symmetric MPE. Section 6 constructs asymmetric equilibria in which players take turns playing the risky arm before all experimentation stops. Section 7 studies two-player equilibria that reward the last experimenter. Section 8 contains concluding remarks. Proofs for all of the corollaries and for Proposition 7 are relegated to the Appendix.

\section{Poisson BANDits}

The setup of the model is similar to that of Keller et al. (2005), the principal difference being that here a bad risky arm yields positive payoffs (as opposed to zero), which means that a success does not reveal the risky arm to be good. For mathematical details on Poisson bandits, see Presman (1990) or Presman and Sonin (1990); for the optimal control of piecewise deterministic processes more broadly, see Davis (1993).

Time $t \in[0, \infty)$ is continuous, and the discount rate is $r>0$. There are $N \geq 1$ players, each endowed with one unit of a perfectly divisible resource per unit of time and each facing a two-armed bandit problem. Lump-sum rewards on the risky arm $R$ are independent draws from a time-invariant distribution on $\mathbb{R} \backslash\{0\}$ with a known mean $h>0$. If a player allocates the fraction $k_{t} \in[0,1]$ of her resource to $R$ over an interval of time $\left[t, t+d t\right.$ [ and, consequently, the fraction $1-k_{t}$ to the safe $\operatorname{arm} S$, then she receives the expected payoff $\left(1-k_{t}\right) s d t$ from $S$, where $s>0$ is a constant known to all players. The probability that she receives a lump-sum payoff from $R$ at some point in the interval is $k_{t} \lambda_{\theta} d t$, where $\theta=1$ if $R$ is good, $\theta=0$ if $R$ is bad, and $\lambda_{1}>\lambda_{0}>0$ are constants known to all players. Therefore, the overall expected payoff increment conditional on $\theta$ is [ $\left.\left(1-k_{t}\right) s+k_{t} \lambda_{\theta} h\right] d t$. We assume that $\lambda_{0} h<s<\lambda_{1} h$, so each player prefers $R$ to $S$ if $R$ is good and prefers $S$ to $R$ if $R$ is bad.

However, players do not know whether the risky arm is good or bad; they start with a common prior belief about $\theta$. Thereafter, all players observe each other's actions and outcomes, so they hold common posterior beliefs throughout time. With $p_{t}$ denoting the subjective probability at time $t$ that players assign to the risky arm being good, a player's expected payoff increment conditional on all available information is [ $\left(1-k_{t}\right) s+$ $\left.k_{t} \lambda\left(p_{t}\right) h\right] d t$ with

$$
\lambda(p)=p \lambda_{1}+(1-p) \lambda_{0}
$$


Given a player's actions $\left\{k_{t}\right\}_{t \geq 0}$ such that $k_{t}$ is measurable with respect to the information available at time $t$, her total expected discounted payoff, expressed in per-period units, is

$$
\mathrm{E}\left[\int_{0}^{\infty} r e^{-r t}\left[\left(1-k_{t}\right) s+k_{t} \lambda\left(p_{t}\right) h\right] d t\right]
$$

where the expectation is over the stochastic processes $\left\{k_{t}\right\}$ and $\left\{p_{t}\right\}$. We note that a player's payoff depends on others' actions only through their effect on the evolution of beliefs, which constitute a natural state variable.

To derive the law of motion of beliefs, suppose that over the interval of time $[t, t+\Delta t[$, player $n$ allocates the constant fraction $k_{n, t}$ of her resource to her risky arm. The sum $K_{t}=\sum_{n=1}^{N} k_{n, t}$ measures how much of the overall resource is allocated to risky arms; we call this number the intensity of experimentation. Conditional on the type of the risky arm, the arrival of lump sums is independent across players. If the risky arms are good, the probability of none of the players receiving a lump-sum payoff is $e^{-K_{t} \lambda_{1} \Delta t}$, and if they are bad, this probability is $e^{-K_{t} \lambda_{0} \Delta t}$. Therefore, given no lump-sum payoff arriving in $[t, t+\Delta t[$, the belief at the end of that time period is

$$
p_{t+\Delta t}=\frac{p_{t} e^{-K_{t} \lambda_{1} \Delta t}}{\left(1-p_{t}\right) e^{-K_{t} \lambda_{0} \Delta t}+p_{t} e^{-K_{t} \lambda_{1} \Delta t}}
$$

by Bayes' rule. As long as no lump sum arrives, the belief thus evolves smoothly with infinitesimal increment $d p_{t}=-K_{t} \Delta \lambda p_{t}\left(1-p_{t}\right) d t$, where $\Delta \lambda=\lambda_{1}-\lambda_{0}$. However, if any of the players receives a lump sum at time $t$, the belief jumps up from $p_{t-}$ (the limit of beliefs held before the arrival of the lump sum) to

$$
p_{t}=\lim _{\Delta t \downarrow 0} \frac{p_{t-}\left[1-e^{-K_{t} \lambda_{1} \Delta t}\right]}{\left(1-p_{t-}\right)\left[1-e^{-K_{t} \lambda_{0} \Delta t}\right]+p_{t-}\left[1-e^{-K_{t} \lambda_{1} \Delta t}\right]}=\frac{\lambda_{1} p_{t-}}{\lambda\left(p_{t-}\right)},
$$

which is independent of the intensity of experimentation. We write

$$
j(p)=\frac{\lambda_{1} p}{\lambda(p)}
$$

for the function that describes beliefs after a success on a risky arm.

We restrict players to Markovian strategies $k_{n}:[0,1] \rightarrow[0,1]$ with the left limit belief $p_{t-}$ as the state variable, so that the action player $n$ takes at time $t$ is $k_{n}\left(p_{t-}\right) .{ }^{4}$ We impose the following restrictions on these strategies: (i) $k_{n}$ is left-continuous; (ii) there is a finite partition of [0,1] into intervals of positive length on each of which $k_{n}$ is Lipschitzcontinuous. By standard results, each profile $\left(k_{1}, k_{2}, \ldots, k_{N}\right)$ of such strategies induces a well defined law of motion for players' common beliefs and well defined payoff functions. A simple strategy is one that takes values in $\{0,1\}$ only, meaning that the player uses one arm exclusively at any given point in time. Finally, a strategy $k_{n}$ is a cutoff strategy if there is a belief $\hat{p}$ such that $k_{n}(p)=1$ for all $p>\hat{p}$ and $k_{n}(p)=0$ otherwise.

\footnotetext{
${ }^{4}$ By definition, $p_{0-}=p_{0}$. Note that $p_{t-}=p_{t}$ at almost all $t$. Working with $p_{t-}$ instead of $p_{t}$ merely enforces the informational restriction that the action taken at time $t$ cannot be conditioned on the arrival of a lump sum at that time.
} 
As a benchmark, a myopic agent simply weighs the short-run payoff from playing the safe arm, $s$, against what he expects from playing the risky arm, $\lambda(p) h$. So he uses the cutoff belief

$$
p^{m}=\frac{s-\lambda_{0} h}{\Delta \lambda h},
$$

playing $R$ for $p>p^{m}$ and $S$ for $p \leq p^{m}$.

\section{JOINT MAXIMIZATION OF AVERAGE PAYOFFS}

Consider $N$ players jointly maximizing their average expected payoff. By the same arguments as in Keller et al. (2005), the value function for the cooperative, expressed as average payoff per agent, satisfies the Bellman equation

$$
u(p)=s+\max _{K \in[0, N]} K\{b(p, u)-c(p) / N\},
$$

where $K$ is the intensity of experimentation,

$$
c(p)=s-\lambda(p) h
$$

is the opportunity cost of playing $R$, and

$$
b(p, u)=\left[\lambda(p)(u(j(p))-u(p))-\Delta \lambda p(1-p) u^{\prime}(p)\right] / r
$$

is the expected benefit of playing $R$. The latter has two parts: a discrete improvement in the overall payoff after a success and a marginal decrease otherwise. ${ }^{5}$

If the shared opportunity cost of playing $R$ exceeds the full expected benefit, the optimal choice is $K=0$ (all agents use $S$ exclusively) and $u(p)=s$. Otherwise, $K=N$ is optimal (all agents use $R$ exclusively) and $u$ satisfies the first-order ordinary differentialdifference equation (henceforth ODDE)

$$
\Delta \lambda p(1-p) u^{\prime}(p)-\lambda(p)[u(j(p))-u(p)]+\frac{r}{N} u(p)=\frac{r}{N} \lambda(p) h
$$

A particular solution to this equation is $u(p)=\lambda(p) h$, the expected per capita payoff from all agents using the risky arm forever.

The option value of being able to change to the safe arm is then captured by the solution to the homogeneous equation, for which we try $u_{0}(p)=(1-p) \Omega(p)^{\mu}$ for some $\mu>0$ to be determined, where

$$
\Omega(p)=\frac{1-p}{p}
$$

\footnotetext{
${ }^{5}$ Infinitesimal changes of the belief are always downward, so it is, in fact, the left-hand derivative of the value function that matters here. This observation will turn out to be of relevance in asymmetric equilibria of the strategic experimentation game.
} 
is the odds ratio. ${ }^{6}$ Now,

$$
u_{0}^{\prime}(p)=-\frac{\mu+p}{p(1-p)} u_{0}(p) \quad \text { and } \quad u_{0}(j(p))=\frac{\lambda_{0}}{\lambda(p)}\left(\frac{\lambda_{0}}{\lambda_{1}}\right)^{\mu} u_{0}(p) .
$$

Inserting these into the homogeneous equation and simplifying leads to the requirement that

$$
\frac{r}{N}+\lambda_{0}-\mu \Delta \lambda=\lambda_{0}\left(\frac{\lambda_{0}}{\lambda_{1}}\right)^{\mu}
$$

As a function of $\mu$, the left-hand side of (2) is a negatively sloped straight line that cuts the vertical axis at $r / N+\lambda_{0}$. The right-hand side is a decreasing exponential function which tends to 0 as $\mu \rightarrow+\infty$, tends to $\infty$ as $\mu \rightarrow-\infty$, and cuts the vertical axis at $\lambda_{0}$. Thus the above equation in $\mu$ has two solutions, one positive and one negative; we write $\mu_{N}$ for the positive solution, which obviously lies between $r /(N \Delta \lambda)$ (the value of $\mu$ where the left-hand side of (2) equals $\lambda_{0}$ ) and $r /(N \Delta \lambda)+\lambda_{0} / \Delta \lambda$ (the value of $\mu$ where it equals 0 ). As the left-hand side of (2) rises with $r / N$, we also see that $\mu_{N}$ is increasing in the discount rate and decreasing in the number of agents.

The solution to the ODDE for $K=N$ is thus

$$
V_{N}(p)=\lambda(p) h+C(1-p) \Omega(p)^{\mu_{N}},
$$

where $C$ is a constant of integration. ${ }^{7}$

Proposition 1 (Cooperative solution). In the $N$-agent cooperative problem, there is a cutoff belief $p_{N}^{*}<p^{m}$ given by

$$
p_{N}^{*}=\frac{\mu_{N}\left(s-\lambda_{0} h\right)}{\left(\mu_{N}+1\right)\left(\lambda_{1} h-s\right)+\mu_{N}\left(s-\lambda_{0} h\right)}
$$

such that below the cutoff it is optimal for all to play $S$ exclusively and above the cutoff it is optimal for all to play $R$ exclusively. The value function $V_{N}^{*}$ for the $N$-agent cooperative is given by

$$
V_{N}^{*}(p)=\lambda(p) h+c\left(p_{N}^{*}\right)\left(\frac{1-p}{1-p_{N}^{*}}\right)\left(\frac{\Omega(p)}{\Omega\left(p_{N}^{*}\right)}\right)^{\mu_{N}}
$$

when $p>p_{N}^{*}$ and by $V_{N}^{*}(p)=s$ otherwise.

\footnotetext{
${ }^{6}$ This guess can be obtained by extrapolation from the limiting case $\lambda_{0}=0$ studied in Keller et al. (2005). In this case, $j(p)=1$ and $u(j(p))=\lambda_{1} h$, so (1) becomes a linear differential equation; the above function $u_{0}$ is easily seen to solve the corresponding homogeneous equation for $\mu=r /\left(N \lambda_{1}\right)$. A more systematic approach is to write the solution of the homogeneous equation as $1-p$ times some unknown function, reflecting the fact that the option to switch from $R$ to $S$ is valuable only if the risky arm is bad. After a change of the independent variable from $p$ to $\ln \Omega(p)$, this unknown function then solves a linear ODDE with constant coefficients and constant delay to which results from Bellman and Cooke (1963) can be applied.

${ }^{7}$ The planner's solution in Bolton and Harris (1999) has the same structure. Only the expression for the expected current payoff from a risky arm and the exponent of the odds ratio differ across setups. Cohen and Solan (2009) show that this continues to be true when the risky arm generates payoffs according to a Lévy process (that is, a continuous-time process with stationary independent increments) with a binary prior on its characteristics.
} 
Proof. The expression for $p_{N}^{*}$ and the constant of integration in (5) are obtained by imposing $V_{N}^{*}\left(p_{N}^{*}\right)=s$ (value matching) and $\left(V_{N}^{*}\right)^{\prime}\left(p_{N}^{*}\right)=0$ (smooth pasting). Then $b\left(p, V_{N}^{*}\right)$ falls short of $c(p) / N$ to the left of $p_{N}^{*}$, coincides with it at $p_{N}^{*}$, and exceeds it to the right of $p_{N}^{*}$. So $V_{N}^{*}$ solves the Bellman equation, with the maximum being achieved at the intensity of experimentation stated in the proposition.

The above proposition determines the efficient strategies. As in Bolton and Harris (1999) and Keller et al. (2005), it is efficient to use a common cutoff strategy; the cutoff increases in $s$ and $\mu_{N}$ (and hence in $r / N$ ). The efficient intensity of experimentation exhibits a bang-bang feature, being maximal when the current belief is above $p_{N}^{*}$ and minimal when it is below.

\section{The strategic Problem}

From now on, we assume that there are $N \geq 2$ players acting noncooperatively. Our solution concept is Markov perfect equilibrium with the common belief as the state variable.

With $K_{\neg n}(p)=\sum_{\ell \neq n} k_{\ell}(p)$, and $b\left(p, u_{n}\right)$ and $c(p)$ as defined in Section 3 above, player $n$ 's payoff function $u_{n}$ is continuous, piecewise differentiable, and satisfies

$$
u_{n}(p)=s+K_{\neg n}(p) b\left(p, u_{n}\right)+k_{n}(p)\left\{b\left(p, u_{n}\right)-c(p)\right\}
$$

on $[0,1]$, with the second term on the right-hand side measuring the benefit of the information generated by the other players.

A strategy $k_{n}^{*}$ for player $n$ is a best response against his opponents' strategies if and only if the resulting payoff function $u_{n}$ solves the Bellman equation

$$
u_{n}(p)=s+K_{\neg n}(p) b\left(p, u_{n}\right)+\max _{k_{n} \in[0,1]} k_{n}\left\{b\left(p, u_{n}\right)-c(p)\right\}
$$

on [0,1], and $k_{n}^{*}(p)$ achieves the maximum on the right-hand side at each belief $p$. It is straightforward to show that if player $n$ plays a best response, his benefit of experimentation $b\left(p, u_{n}\right)$ is nonnegative at all beliefs, and his payoff function $u_{n}$ is nondecreasing in the other players' experimentation schedule $K_{\neg n}$. Standard results further imply that a best-response payoff function $u_{n}$ is once continuously differentiable at any point of continuity of $K_{\neg n}$.

At the boundaries of the unit interval, the obvious optimal actions are $k_{n}^{*}(0)=0$ and $k_{n}^{*}(1)=1$, which implies $u_{n}(0)=s$ and $u_{n}(1)=\lambda_{1} h$ for the player's payoff function. More generally, player $n$ 's best response is obtained by comparing the opportunity cost of playing $R$ with the expected private benefit. If $c(p)>b\left(p, u_{n}\right)$, then $k_{n}^{*}(p)=0$, and the Bellman equation implies $u_{n}(p)=s+K_{\neg n}(p) b\left(p, u_{n}\right)<s+K_{\neg n}(p) c(p)$. If $c(p)=$ $b\left(p, u_{n}\right)$, then $k_{n}^{*}(p)$ is arbitrary in [0,1], and $u_{n}(p)=s+K_{\neg n}(p) c(p)$. Finally, if $c(p)<$ $b\left(p, u_{n}\right)$, then $k_{n}^{*}(p)=1$, and $u_{n}(p)=s+\left(K_{\neg n}(p)+1\right) b\left(p, u_{n}\right)-c(p)>s+K_{\neg n}(p) c(p)$. Thus, exactly as in Keller et al. (2005), player $n$ 's best response to a given intensity of experimentation $K_{\neg n}$ depends on whether in the $(p, u)$ plane, the point $\left(p, u_{n}(p)\right)$ lies below, on, or above the line

$$
\mathcal{D}_{K_{\neg n}}=\left\{(p, u) \in[0,1] \times \mathbb{R}_{+} \mid u=s+K_{\neg n} c(p)\right\} .
$$


For $K_{\neg n}>0$ this is a downward sloping diagonal that cuts the safe payoff line $u=s$ at the myopic cutoff $p^{m}$; for $K_{\neg n}=0$, it coincides with the safe payoff line.

The following two observations also carry over verbatim from Keller et al. (2005). First, no profile of Markov strategies can generate an average payoff that exceeds $V_{N}^{*}$, and the payoff of a player using a best response to her opponents' strategies cannot fall below $V_{1}^{*}$. The upper bound follows immediately from the fact that the cooperative solution maximizes the average payoff. The intuition for the lower bound is that an agent can only benefit from the information generated by others. Second, all Markov perfect equilibria are inefficient. Along the efficient experimentation path, the benefit of an additional experiment tends to $1 / N$ of its opportunity cost as $p$ approaches $p_{N}^{*}$. A self-interested player compares the benefit of an additional experiment with the full opportunity cost and so has an incentive to deviate from the efficient path by using $S$ instead of $R$.

It is also easy to see that in any Markov perfect equilibrium, the set of beliefs at which the intensity of experimentation is positive must be an interval, and that it must contain the half-open interval $\left.] p_{1}^{*}, 1\right]$, where $p_{1}^{*}$ is the single-agent cutoff. The interesting question is whether experimentation continues below $p_{1}^{*}$, i.e., whether there is an encouragement effect. Bolton and Harris (1999) show that the encouragement effect is present in the symmetric Markov perfect equilibrium of their model. The next result shows that all MPE of our model exhibit the encouragement effect.

Proposition 2 (Encouragement effect). In any Markov perfect equilibrium, at least one player experiments at some beliefs below $p_{1}^{*}$.

Proof. Suppose to the contrary that all players play $S$ at all beliefs $p \leq p_{1}^{*}$. Then each player's payoff function satisfies $u_{n}\left(p_{1}^{*}\right)=s$ with the left-hand derivative $u_{n}^{\prime}\left(p_{1}^{*}-\right)=0$. For $S$ to be optimal, we must have $b\left(p_{1}^{*}, u_{n}\right) \leq c\left(p_{1}^{*}\right)=b\left(p_{1}^{*}, V_{1}^{*}\right)$, and hence $u_{n}\left(j\left(p_{1}^{*}\right)\right) \leq$ $V_{1}^{*}\left(j\left(p_{1}^{*}\right)\right)$, which must in fact hold as an equality. Thus, the difference $u_{n}-V_{1}^{*}$ assumes its minimum (of 0) at $j\left(p_{1}^{*}\right)$, which implies $u_{n}^{\prime}\left(j\left(p_{1}^{*}\right)-\right) \leq\left(V_{1}^{*}\right)^{\prime}\left(j\left(p_{1}^{*}\right)-\right)$. As $u_{n}\left(j^{2}\left(p_{1}^{*}\right)\right) \geq$ $V_{1}^{*}\left(j^{2}\left(p_{1}^{*}\right)\right)$, this implies $b\left(j\left(p_{1}^{*}\right), u_{n}\right) \geq b\left(j\left(p_{1}^{*}\right), V_{1}^{*}\right)$ and hence $b\left(j\left(p_{1}^{*}\right), u_{n}\right)>c\left(j\left(p_{1}^{*}\right)\right)$. So all players must use $R$ at the belief $j\left(p_{1}^{*}\right)$. By the ODDE for $V_{1}^{*}$ and the explicit solution in Proposition 1 , we have $b\left(j\left(p_{1}^{*}\right), V_{1}^{*}\right)=V_{1}^{*}\left(j\left(p_{1}^{*}\right)\right)-s+c\left(j\left(p_{1}^{*}\right)\right)=V_{1}^{*}\left(j\left(p_{1}^{*}\right)\right)-$ $\lambda\left(j\left(p_{1}^{*}\right)\right) h>0$. Each player's Bellman equation now yields

$$
\begin{aligned}
u_{n}\left(j\left(p_{1}^{*}\right)\right) & =s+N b\left(j\left(p_{1}^{*}\right), u_{n}\right)-c\left(j\left(p_{1}^{*}\right)\right) \\
& \geq s+N b\left(j\left(p_{1}^{*}\right), V_{1}^{*}\right)-c\left(j\left(p_{1}^{*}\right)\right) \\
& >s+b\left(j\left(p_{1}^{*}\right), V_{1}^{*}\right)-c\left(j\left(p_{1}^{*}\right)\right) \\
& =V_{1}^{*}\left(j\left(p_{1}^{*}\right)\right),
\end{aligned}
$$

which contradicts the equality $u_{n}\left(j\left(p_{1}^{*}\right)\right)=V_{1}^{*}\left(j\left(p_{1}^{*}\right)\right)$ derived earlier.

The idea behind the proof is that the only way that all experimentation could stop at $p_{1}^{*}$ is for the "jump benefit" to be the same for each of the $N$ players as for a lone agent, 
given the same opportunity cost and the same "slide disbenefit"; but this would imply that $u_{n}$ and $V_{1}^{*}$ matched in value not only at $p_{1}^{*}$ but also at $j\left(p_{1}^{*}\right)$. This is not possible, since at $j\left(p_{1}^{*}\right)$ the benefit of a further jump up is no less and the disbenefit of a slide down is no worse for player $n$ than for a lone agent, and if a lone agent has an incentive to experiment, then so do each of the $N$ players-the positive externality resulting in a higher value at $j\left(p_{1}^{*}\right)$.

Our second general result on Markov perfect equilibria concerns the nonexistence of equilibria where all players use cutoff strategies.

Proposition 3 (No MPE in cutoff strategies). In any Markov perfect equilibrium, at least one player uses a strategy that is not of the cutoff type.

Proof. Suppose to the contrary that there is an MPE where all players use a cutoff strategy. For $n=1, \ldots, N$, let $p_{n}$ denote the belief at which player $n$ switches from using $R$ exclusively to using $S$ exclusively. Clearly, $p_{n} \leq p^{m}$ for all $n$. Without loss of generality, we can assume that $p_{1} \leq p_{2} \leq \cdots \leq p_{N-1} \leq p_{N}$. Moreover, we must have $p_{1}<p^{m}$, since each player would have an incentive to deviate to the optimal strategy of a single player otherwise.

Suppose that $p_{1}=p_{2}$. Immediately to the right of this cutoff, both $u_{1}$ and $u_{2}$ must then lie below $\mathcal{D}_{1}$, so players 1 and 2 playing $R$ are not best responses. This proves that $p_{1}<p_{2}$.

Now, $u_{2}$ must lie below $\mathcal{D}_{1}$ immediately to the left of $p_{2}$ (as player 2 finds it optimal to free-ride on one opponent who plays $R$ ) and above $\mathcal{D}_{1}$ immediately to the right of $p_{2}$ (as player 2 finds it optimal to join in with at least one opponent who plays $R$ ), so $u_{2}$ crosses $\mathcal{D}_{1}$ at $p_{2}$. (In fact, one can iterate this argument to establish that all cutoffs are different, and that $u_{n}$ crosses $\mathcal{D}_{n-1}$ at $p_{n}$.)

Since a player's payoff function is weakly increasing in the intensity of experimentation provided by the other players, we have $u_{1} \leq u_{2}$, and so $u_{1}$ is either below or exactly on $\mathcal{D}_{1}$ at $p_{2}$. In the first case, there is an interval ] $p_{2}, p_{2}+\epsilon$ [, where player 1 (who is assumed to play $R$ ) is not responding optimally to the other players' combined intensity of experimentation $K_{\neg 1}=1$. In the second case, $u_{1}=u_{2}$ on $\left[p_{2}, 1\right]$ and $u_{1}^{\prime}\left(p_{2}-\right) \geq u_{2}^{\prime}\left(p_{2}-\right)$, hence $b\left(p_{2}, u_{1}\right) \leq b\left(p_{2}, u_{2}\right)$. But then, $u_{2}\left(p_{2}\right)=s+b\left(p_{2}, u_{2}\right)>s+b\left(p_{2}, u_{1}\right)-c\left(p_{2}\right)=$ $u_{1}\left(p_{2}\right)$, a contradiction.

We now turn to a detailed investigation of Markov perfect equilibria.

\section{Symmetric EQUilibrium}

In view of Proposition 3, a symmetric MPE necessarily requires the players to choose an interior allocation of their resource at some beliefs. When this happens, the indifference condition $b(p, u)=c(p)$ implies that the common payoff function solves the ODDE

$$
\Delta \lambda p(1-p) u^{\prime}(p)-\lambda(p)[u(j(p))-u(p)]=r \lambda(p) h-r s .
$$


Otherwise, either all players play $S$ exclusively and the common payoff is $u(p)=s$ or all players play $R$ exclusively and the common payoff function $u$ satisfies (1), hence is of the form $V_{N}$ given in (3).

In the $(p, u)$ plane, the region where all players use the risky arm exclusively and the region where they use both arms simultaneously are separated by the diagonal $\mathcal{D}_{N-1}$. Given the post-jump value $u(j(p))$, we have smooth pasting of the solutions to (1) and (6) along $\mathcal{D}_{N-1}$. Smooth pasting also occurs at the boundary of the region where all players use $S$ exclusively with the region where they use both arms. In other words, $u$ must be of class $C^{1}$. To see this, suppose we had a symmetric equilibrium with a payoff function that hits the level $s$ at the belief $\tilde{p}$ with slope $u^{\prime}(\tilde{p}+)>0$. Then, at beliefs immediately to the right of $\tilde{p}$, we would have $b(p, u)=c(p)$ or

$$
\lambda(p)[u(j(p))-u(p)] / r=c(p)+\Delta \lambda p(1-p) u^{\prime}(p) / r,
$$

implying

$$
\lambda(\tilde{p})[u(j(\tilde{p}))-s] / r=c(\tilde{p})+\Delta \lambda \tilde{p}(1-\tilde{p}) u^{\prime}(\tilde{p}+) / r>c(\tilde{p})
$$

by continuity. Immediately to the left of $\tilde{p}$, continuity of $u(j(p))$ and the fact that $u^{\prime}(p)=0$ would then imply $b(p, u)=\lambda(p)[u(j(p))-s] / r>c(p)$, so there would be an incentive to deviate from $S$ to $R$.

Proposition 4 (Symmetric equilibrium). The $N$-player experimentation game has a unique symmetric Markov perfect equilibrium with the common posterior belief as the state variable. The corresponding payoff function is the unique function $W_{N}:[0,1] \rightarrow$ $\left[s, \lambda_{1} h\right]$ of class $C^{1}$ with the following properties: $W_{N}(p)=s$ on an interval $\left[0, \tilde{p}_{N}\right]$ with $p_{N}^{*}<\tilde{p}_{N}<p_{1}^{*} ; W_{N}(p)>s$ on $\left.] \tilde{p}_{N}, 1\right] ; W_{N}$ solves (6) on an interval $] \tilde{p}_{N}, p_{N}^{\dagger}[$ with $\tilde{p}_{N}<p_{N}^{\dagger}<p^{m}$ and solves (1) on ] $p_{N}^{\dagger}, 1$ [. The players' common equilibrium strategy is continuous in the posterior belief and satisfies $k(p)=0$ for $p \leq \tilde{p}_{N}$,

$$
\left.k(p)=\frac{1}{N-1} \frac{W_{N}(p)-s}{c(p)} \in\right] 0,1[
$$

for $\tilde{p}_{N}<p<p_{N}^{\dagger}$, and $k(p)=1$ for $p \geq p_{N}^{\dagger}$. The payoff function $W_{N}$ is increasing on $\left[\tilde{p}_{N}, 1\right]$ and the strategy $k$ is increasing on $\left[\tilde{p}_{N}, p_{N}^{\dagger}\right]$.

Proof. We first show that there is at most one symmetric MPE. Suppose that we have two symmetric equilibria with different payoff functions $u$ and $\hat{u}$, respectively, both of which must be of class $C^{1}$. Let $u-\hat{u}$ assume a negative global minimum at the belief $p$, which by necessity must lie in the open unit interval. At this belief, $u^{\prime}(p)=\hat{u}^{\prime}(p)$ and $u(j(p))-\hat{u}(j(p)) \geq u(p)-\hat{u}(p)$, so $b(p, u) \geq b(p, \hat{u})$. We cannot have both $u(p)$ and $\hat{u}(p)$ above $\mathcal{D}_{N-1}$, since in this region both $u$ and $\hat{u}$ are of the form (3) and the difference $u-\hat{u}$ is increasing to the right of $\mathcal{D}_{N-1}$. Further, if $\hat{u}(p)$ is above $\mathcal{D}_{N-1}$ and $u(p)$ is on or below, then $b(p, \hat{u})>c(p)=b(p, u)$ in contradiction to what we derived before. Consequently, we must have both $\hat{u}(p)$ and $u(p)$ on or below $\mathcal{D}_{N-1}$, so $b(p, \hat{u})=c(p)=b(p, u)$. This in turn yields $u(j(p))-\hat{u}(j(p))=u(p)-\hat{u}(p)$, so the difference $u-\hat{u}$ is also at its minimum at the belief $j(p)$. Iterating the argument until we 
get to the right of $p^{m}$ (and hence to the right of $\mathcal{D}_{N-1}$ ), we obtain another contradiction, which proves that $u \geq \hat{u}$. By the same arguments, $\hat{u}-u$ cannot assume a negative global minimum either, and so $u=\hat{u}$.

Next, we sketch the construction of the symmetric equilibrium; for details, see the Appendix. Varying the point of intersection with the diagonal $\mathcal{D}_{N-1}$, one first constructs a family of candidate value functions that solve the ODDE (1) ( $N$ players using $R$ exclusively) above $\mathcal{D}_{N-1}$ and the ODDE (6) (indifference between $R$ and $S$ ) below. Using an intermediate-value argument, we then establish the existence of one such function that reaches the level $s$ with zero slope as we move down from $p=p^{m}$ to lower beliefs. This function is easily seen to solve each player's Bellman equation. Finally, the identity $u_{n}(p)=s+K_{\neg n}(p) c(p)$ uniquely determines the common intensity of experimentation in the range of beliefs where the value function lies below $\mathcal{D}_{N-1}$ but above the level $s$.

We can represent the equilibrium payoff function $W_{N}$ in closed form up to some constants of integration that are implicitly determined by $p_{N}^{\dagger}$.

Corollary 1. Define intervals $J_{0}=\left[p_{N}^{\dagger}, 1\right]$ and $J_{i}=\left[j^{-i}\left(p_{N}^{\dagger}\right), j^{-(i-1)}\left(p_{N}^{\dagger}\right)[\right.$ for $i=$ $1,2, \ldots$. If $\mu_{N} \neq \lambda_{0} / \Delta \lambda,{ }^{8}$ then

$$
\begin{aligned}
W_{N}(p)=\lambda( & p) h+i\left[\frac{r}{\lambda_{1}}\left(\lambda_{1} h-s\right) p-\frac{r}{\lambda_{0}}\left(s-\lambda_{0} h\right)(1-p)\right] \\
& +C^{(0)}\left(\frac{\lambda_{0}\left(\lambda_{0} / \lambda_{1}\right)^{\mu_{N}}}{\lambda_{0}-\mu_{N} \Delta \lambda}\right)^{i}(1-p) \Omega(p)^{\mu_{N}} \\
& +\sum_{\eta=0}^{i-1} \frac{C^{(i-\eta)}}{\eta !}\left(-\frac{\lambda_{0}\left(\lambda_{0} / \lambda_{1}\right)^{\lambda_{0} / \Delta \lambda}}{\Delta \lambda} \ln \left[\left(\lambda_{0} / \lambda_{1}\right)^{\eta-1} \Omega(p)\right]\right)^{\eta}(1-p) \Omega(p)^{\lambda_{0} / \Delta \lambda}
\end{aligned}
$$

on $J_{i} \cap\left\{p \mid W_{N}(p)>s\right\}$ for some constants $C^{(i-\eta)}(\eta=0, \ldots, i-1)$, chosen to ensure continuity of $W_{N}$. The constant $C^{(0)}$ ensuring that $\left(p_{N}^{\dagger}, W_{N}\left(p_{N}^{\dagger}\right)\right) \in \mathcal{D}_{N-1}$ is given by

$$
C^{(0)}=N c\left(p_{N}^{\dagger}\right)\left(1-p_{N}^{\dagger}\right)^{-1} \Omega\left(p_{N}^{\dagger}\right)^{-\mu_{N}} .
$$

The proof (given in the Appendix) shows how the constants $C^{(i)}$ can be calculated recursively given $C^{(0)}$.

Figure 2 in Section 6 below depicts the intensity of experimentation in the symmetric equilibrium with two players (rightmost solid curve). The dotted step function is the efficient intensity.

The symmetric equilibrium of the Poisson model shares the main features with its counterpart in the Brownian model of Bolton and Harris (1999). First, because of the incentive to free-ride, experimentation stops for good inefficiently early (the lower threshold $\tilde{p}_{N}$ is above the cooperative cutoff $p_{N}^{*}$ ), and the intensity of experimentation is inefficiently low at any belief between $p_{N}^{*}$ and $p_{N}^{\dagger}$. Second, there is the encouragement effect ( $\tilde{p}_{N}$ is below the single-agent cutoff $p_{1}^{*}$ ). Third, both the incentive to free-ride and the

\footnotetext{
${ }^{8}$ The proof makes it obvious how to modify this result in the knife-edge case where $\mu_{N}=\lambda_{0} / \Delta \lambda$.
} 
encouragement effect become stronger as the number of players increases. ${ }^{9}$ Fourth, the acquisition of information is slowed down so severely near $\tilde{p}_{N}$ that the players' beliefs cannot reach this threshold in finite time.

CoRollary 2. Starting from a prior belief above $\tilde{p}_{N}$, the players' common posterior belief never reaches this threshold in the symmetric Markov perfect equilibrium.

This result strongly suggests that asymmetric equilibria where the rate of information acquisition stays bounded away from zero before all experimentation ceases ought to be more efficient than symmetric equilibrium. The next section confirms this.

\section{Asymmetric Equilibria}

Our construction of asymmetric Markov perfect equilibria rests on two ideas. The first is to give the players a common continuation value after any success on a risky arm; this allows us to construct the players' average payoff function before assigning individual strategies. The second is to let them alternate between the roles of experimenter and free-rider before all experimentation stops; this allows us to achieve an overall intensity of experimentation higher than in the symmetric equilibrium, yielding higher equilibrium payoffs.

In fact, for points $(p, u)$ below the diagonal $\mathcal{D}_{1-1 / N}$, the common action that keeps all players indifferent between $R$ and $S$, and gives them $u$ as the common continuation value, $k=(u-s) /[(N-1) c(p)]$, implies an intensity of experimentation $K=N k<1$. In contrast, the equilibria we construct have $K=1$ over some range of beliefs where the graph of the average payoff function lies below $\mathcal{D}_{1-1 / N}$. We achieve this by partitioning the range in question into a finite number of intervals, on each of which exactly one player plays risky.

If the last of these "lone experimenters" stops using the risky arm at the belief $\bar{p}$, his value function $u$ satisfies $\lambda(\bar{p})[u(j(\bar{p}))-s] / r=c(\bar{p})$ as the left derivative $u^{\prime}(\bar{p})=0$. When all players have a common continuation value after a success on a risky arm, this equation also holds for the players' average payoff function $\bar{u}$ and so $\lambda(\bar{p}) \times$ $[\bar{u}(j(\bar{p}))-s] / r=c(\bar{p})$. Varying $\bar{p}$, we can trace out the locus $\overline{\mathcal{D}}$ of all possible post-jump points $(j(\bar{p}), \bar{u}(j(\bar{p})))$ in the $(p, u)$ plane that satisfy this condition:

$$
\overline{\mathcal{D}}=\left\{(p, u) \in[0,1] \times \mathbb{R}_{+} \mid \lambda\left(j^{-1}(p)\right)[u-s] / r=c\left(j^{-1}(p)\right)\right\} .
$$

Using the fact that $\lambda\left(j^{-1}(p)\right)=\lambda_{0} \lambda_{1} /\left[p \lambda_{0}+(1-p) \lambda_{1}\right]$, it is straightforward to show that $\overline{\mathcal{D}}$ is a downward sloping straight line through the points $\left(0, s+r\left[s-\lambda_{0} h\right] / \lambda_{0}\right)$ and $\left(j\left(p^{m}\right), s\right)$.

To ensure both a common continuation value after any success and an increase in the intensity of experimentation relative to the symmetric MPE, we start our construction of the average equilibrium payoff function at some point $\left(p^{\sharp}, u\right)$ on the lower envelope $\overline{\mathcal{D}} \wedge \mathcal{D}_{1-1 / N}$ of the diagonals $\overline{\mathcal{D}}$ and $\mathcal{D}_{1-1 / N}$. This lower envelope coincides with

\footnotetext{
${ }^{9}$ As $N$ increases, each player obtains a higher payoff at all beliefs where the risky arm is used some of the time, and $\tilde{p}_{N}$ falls. The diagonal $\mathcal{D}_{N-1}$ rotates clockwise, tending to increase $p_{N}^{\dagger}$, but since the payoff function shifts upward, the overall effect on $p_{N}^{\dagger}$ is ambiguous.
} 
$\mathcal{D}_{1-1 / N}$ if and only if $r / \lambda_{0} \geq 1-1 / N$, so $\overline{\mathcal{D}}$ is relevant only for sufficiently high $\lambda_{0}$, that is, for sufficiently small jumps of beliefs after successes. To the right of $p^{\sharp}$, we proceed as in the construction of the symmetric MPE. To the left of $p^{\sharp}$, we solve for the average payoff function when one player out of $N$ is playing risky. Varying $p^{\sharp}$, we then ensure that the average payoff hits the level $s$ at a belief $p^{b}$, where the last experimenter is indeed indifferent between playing risky and playing safe. If the point $\left(p^{\sharp}, u\right)$ thus determined lies below $\overline{\mathcal{D}}$ (and hence on $\mathcal{D}_{1-1 / N}$ ), we have $j\left(p^{\mathrm{b}}\right)>p^{\sharp}$; if this point lies on $\overline{\mathcal{D}}$, we have $j\left(p^{b}\right)=p^{\sharp}$. In either case, a success at any belief to the right of $p^{b}$ makes the belief jump to the right of $p^{\sharp}$, where the equilibrium involves symmetric actions and continuation payoffs that coincide with the average. Between $p^{b}$ and $p^{\sharp}$, moreover, the graph of the average payoff function lies below $\mathcal{D}_{1-1 / N}$, and so an intensity of experimentation equal to 1 is indeed more than would be compatible with symmetric behavior.

For $N=2$, Figure 1 illustrates the payoff functions that can arise in the equilibria we construct and gives the corresponding intensity of experimentation in various regions of the $(p, u)$ plane. The faint straight lines ending in $\left(p^{m}, s\right)$ are the diagonals $\mathcal{D}_{1}$ and $\mathcal{D}_{1 / 2}$; the faint straight line ending on $\mathcal{D}_{1 / 2}$ is the part of $\overline{\mathcal{D}} \wedge \mathcal{D}_{1 / 2}$ that lies below $\mathcal{D}_{1 / 2}$; the solid kinked line is the myopic payoff. The solid curves are the graphs of the players' payoff functions. The equilibrium intensity of experimentation varies along the graph of the average equilibrium payoff function. The intensity is 2 when the graph is above $\mathcal{D}_{1}$, between 1 and 2 when the graph lies between $\mathcal{D}_{1 / 2}$ and $\mathcal{D}_{1}$, etc. The intensity of experimentation is continuous in beliefs at $p^{\sharp}$ if the graph crosses $\overline{\mathcal{D}} \wedge \mathcal{D}_{1 / 2}$ on $\mathcal{D}_{1 / 2}$, as in the figure. If the graph crosses $\overline{\mathcal{D}} \wedge \mathcal{D}_{1 / 2}$ below $\mathcal{D}_{1 / 2}$, the intensity jumps at the belief $p^{\sharp}$.

Figure 2 compares the intensity of experimentation with that in the symmetric twoplayer equilibrium. The dotted step function is the efficient intensity; the rightmost solid curve is the intensity in the symmetric MPE.

For arbitrary $N$, we have the following result.

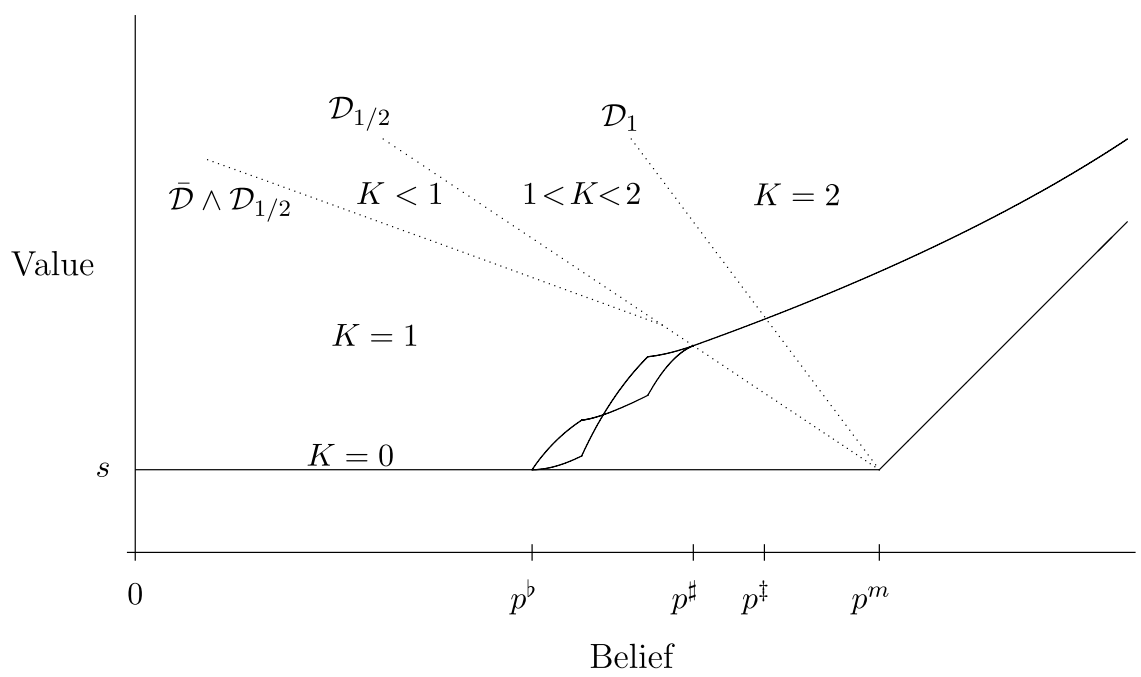

FIgURE 1. Equilibrium payoffs in a two-player asymmetric equilibrium. 


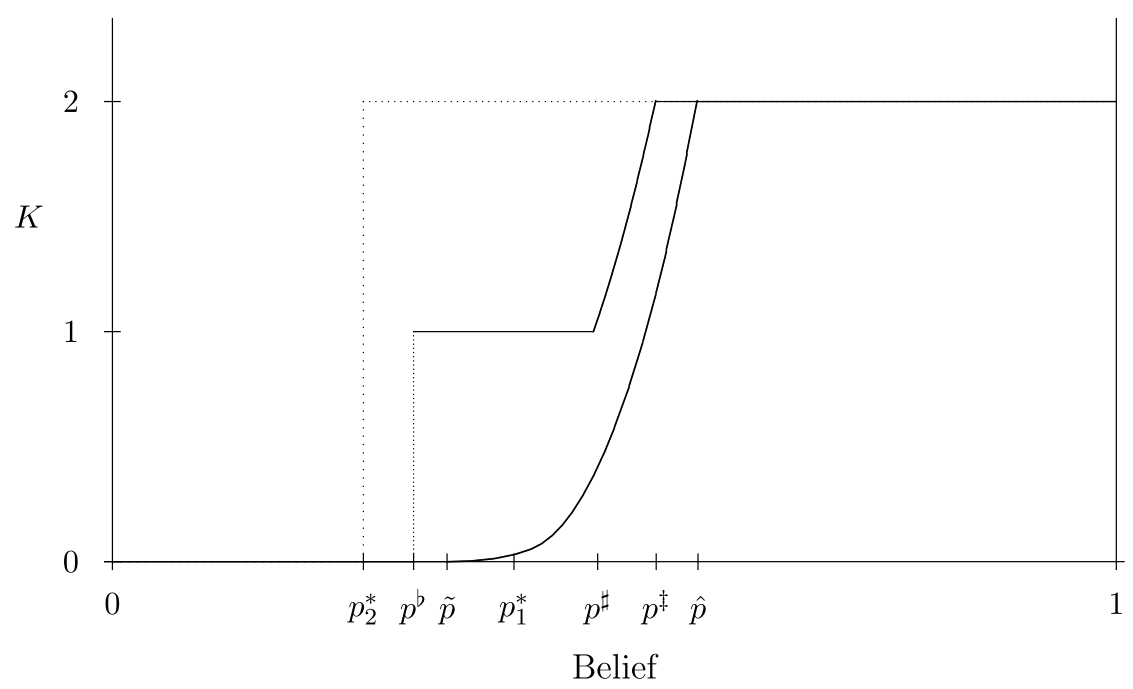

FIGURE 2. Intensity of experimentation in the cooperative solution, the equilibria of Proposition 5 , and the symmetric equilibrium with two players.

Proposition 5 (Asymmetric MPE). The $N$-player experimentation game admits Markov perfect equilibria with three thresholds, $p_{N}^{\mathrm{b}}$, $p_{N}^{\sharp}$, and $p_{N}^{\ddagger}$, where $p_{N}^{*}<p_{N}^{\mathrm{b}}<p_{N}^{\sharp}<p_{N}^{\ddagger}<$ $p^{m}$ and $j\left(p_{N}^{b}\right) \geq p_{N}^{\sharp}$ such that on $\left[p_{N}^{\sharp}, 1\right]$, the players have a common payoff function; on $\left[p_{N}^{\ddagger}, 1\right]$, all players play $R$; on $] p_{N}^{\sharp}, p_{N}^{\ddagger}[$, the players allocate a common interior fraction of the unit resource to $R$, and this fraction increases in the belief; on $\left.] p_{N}^{b}, p_{N}^{\sharp}\right]$, the intensity of experimentation equals 1 with players taking turns playing $R$ on consecutive subintervals; on $\left[0, p_{N}^{b}\right]$, all players play $S$. The intensity of experimentation is continuous in beliefs on ] $\left.p_{N}^{b}, 1\right]$ with the possible exception of a jump at $p_{N}^{\sharp}$. The average payoff function is increasing on $\left[p_{N}^{b}, 1\right]$ and once continuously differentiable on the unit interval except for the beliefs $p_{N}^{b}$ and, if the intensity of experimentation has a jump there, $p_{N}^{\sharp}$. On ]$p_{N}^{b}, 1\left[\right.$, the average payoff is higher than in the symmetric MPE, and $p_{N}^{b}$ lies to the left of the cutoff $\tilde{p}_{N}$ at which all experimentation stops in that equilibrium.

Proof. We just sketch the construction of the equilibrium here; details can be found in the Appendix. First, we construct the players' average payoff function $\bar{u}$ in the purported equilibria, using an approach similar to the proof of Proposition 4. This function is increasing on $\left[p_{N}^{b}, 1\right]$. Its graph crosses $\overline{\mathcal{D}} \wedge \mathcal{D}_{1-1 / N}$ at $p_{N}^{\sharp}$ and $\mathcal{D}_{N-1}$ at $p_{N}^{\ddagger}$. It has a kink at $p_{N}^{\sharp}$ with $\bar{u}^{\prime}\left(p_{N}^{\sharp}-\right)>\bar{u}^{\prime}\left(p_{N}^{\sharp}+\right)$ if and only if the intersection with $\overline{\mathcal{D}} \wedge \mathcal{D}_{1-1 / N}$ is below $\mathcal{D}_{1-1 / N}$. It satisfies $\bar{u}(p)=s+b(p, \bar{u})-c(p) / N$ between $p_{N}^{b}$ and $p_{N}^{\sharp}$, solves the indifference ODDE (6) between $p_{N}^{\sharp}$ and $p_{N}^{\ddagger}$, and is of the form (3) above $p_{N}^{\ddagger}$. The average jump benefit $\lambda\left(p_{N}^{b}\right)\left[\bar{u}\left(j\left(p_{N}^{b}\right)\right)-s\right] / r$ exactly equals the opportunity cost $c\left(p_{N}^{b}\right)$. As all players' payoff functions have a zero left-hand derivative at $p_{N}^{\mathrm{b}}$ and a common value of $\bar{u}\left(j\left(p_{N}^{\mathrm{b}}\right)\right)$ at $j\left(p_{N}^{b}\right)$, each player will, therefore, be indifferent between $R$ and $S$ at $p_{N}^{b}$.

Second, we construct the players' payoff functions and strategies. To this end, we split the interval $\left.] p_{N}^{b}, p_{N}^{\sharp}\right]$ into finitely many subintervals $\left.] p_{\ell, i}, p_{r, i}\right]$ and in turn partition 
each of them into $N$ intervals $I_{1, i}, \ldots, I_{N, i}$. We let player $n$ use $R$ on all intervals $I_{n, i}$ and use $S$ on $\left.] p_{N}^{b}, p_{N}^{\sharp}\right] \backslash \bigcup_{i} I_{n, i}$. Using intermediate-value arguments, we can choose the intervals $I_{1, i}, \ldots, I_{N, i}$ such that each player's payoff function coincides with $\bar{u}$ at the boundaries of each subinterval $\left.] p_{\ell, i}, p_{r, i}\right]$. By increasing the number and reducing the size of these subintervals, moreover, we can ensure that the vertical distance $\left|u_{n}-\bar{u}\right|$ remains below some given real number $\delta>0$ for all $n$.

Third, we verify that for sufficiently small $\delta$, that is, for sufficiently frequent alternation between the roles of free-rider and experimenter on $\left.] p_{N}^{b}, p_{N}^{\sharp}\right]$, the strategies we have constructed are mutually best responses.

As to the comparison of the average payoff function $\bar{u}$ with that of the symmetric equilibrium, $W_{N}$, suppose that $\bar{u}-W_{N}$ assumes a negative global minimum at the belief $p$ in the open unit interval. Note that $\bar{u}$ must be differentiable there, since a kink with $\bar{u}^{\prime}\left(p_{N}^{\sharp}-\right)>\bar{u}^{\prime}\left(p_{N}^{\sharp}+\right)$ is incompatible with even a local minimum of $\bar{u}-W_{N}$ at $p_{N}^{\sharp}$. At the belief $p$, therefore, $W_{N}^{\prime}(p)=\bar{u}^{\prime}(p)$ and $\bar{u}(j(p))-W_{N}(j(p)) \geq \bar{u}(p)-W_{N}(p)$, so $b(p, \bar{u}) \geq b\left(p, W_{N}\right)$. We cannot have both $W_{N}(p)$ and $\bar{u}(p)$ above $\mathcal{D}_{1}$, since in this region both $W_{N}$ and $\bar{u}$ are of the form (3) and so the difference $\bar{u}-W_{N}$ is increasing. Further, if $W_{N}(p)$ is above $\mathcal{D}_{1}$ and $\bar{u}(p)$ is on or below that diagonal, then $b\left(p, W_{N}\right)>c(p) \geq b(p, \bar{u})$, in contradiction to what we derived before (to the left of $p_{N}^{\sharp}, \bar{u}(p)=s+b(p, \bar{u})-c(p) / N<s+(1-1 / N) c(p)$ and hence $\left.b(p, \bar{u})<c(p)\right)$. Consequently, we must have both $W_{N}(p)$ and $\bar{u}(p)$ on or below $\mathcal{D}_{1}$, which translates into $b\left(p, W_{N}\right)=c(p) \geq b(p, \bar{u})$ and hence, by what we saw above, $b\left(p, W_{N}\right)=b(p, \bar{u})$. This in turn yields $\bar{u}(j(p))-W_{N}(j(p))=\bar{u}(p)-W_{N}(p)$, so the difference $\bar{u}-W_{N}$ is also at its minimum at the belief $j(p)$. Iterating the argument until we get to the right of $p^{m}$ (and hence to the right of $\mathcal{D}_{1}$ ), we again obtain a contradiction. This establishes $\bar{u} \geq W_{N}$. Now, if we had $\bar{u}(p)=W_{N}(p)$ at some $\left.p \in\right] p_{N}^{b}, 1\left[\right.$, this would again imply $b(p, \bar{u})=b\left(p, W_{N}\right)$ at a global minimum of $\bar{u}-W_{N}$ and, by the iterative argument just given, lead to another contradiction. This proves that $\bar{u}>W_{N}$ on ] $p_{N}^{b}, 1\left[\right.$. In particular, $p_{N}^{\mathrm{b}} \leq \tilde{p}_{N}$, the belief at which all experimentation stops in the symmetric MPE. The equality $p_{N}^{\mathrm{b}}=\tilde{p}_{N}$ would entail $\lambda\left(p_{N}^{\mathrm{b}}\right)\left[\bar{u}\left(j\left(p_{N}^{\mathrm{b}}\right)\right)-s\right] / r=c\left(p_{N}^{\mathrm{b}}\right)=\lambda\left(p_{N}^{\mathrm{b}}\right)\left[W_{N}\left(j\left(p_{N}^{\mathrm{b}}\right)\right)-s\right] / r$ and hence $\bar{u}\left(j\left(p_{N}^{\mathrm{b}}\right)\right)=$ $W_{N}\left(j\left(p_{N}^{b}\right)\right)$, which we have already shown to be impossible.

The gain in average payoffs relative to the symmetric equilibrium stems from the fact that, owing to the alternation between the roles of lone experimenter and free-rider, the intensity of experimentation is bounded away from zero immediately above the belief where all experimentation stops. In the symmetric equilibrium, a player who deviates to the safe action slows down the gradual slide of beliefs toward more pessimism; as the opponents' strategies are increasing functions of the level of optimism, the deviation causes them to experiment more than they would on the equilibrium path. When players use beliefs to coordinate their alternation between experimentation and freeriding, by contrast, a deviation from the risky to the safe action freezes the belief in its current state and delays the time at which another player takes over the burden of experimentation. Deviations are thus more attractive under symmetric strategies than under alternation. This explains why the equilibrium intensity under the latter can be higher. 
For beliefs above $p_{N}^{\sharp}$, the players' common payoff function permits an explicit representation of the form given in Corollary 1 . For beliefs between $p_{N}^{b}$ and $p_{N}^{\sharp}$, we have the following result.

Corollary 3. For $p \in] p_{N}^{b}, p_{N}^{\sharp}$, let $\iota$ be the smallest integer such that $j^{\imath+1}(p) \geq p_{N}^{\ddagger}$, i.e., $\iota+1$ consecutive successes would result in all the players playing $R$ exclusively. Then, with $k_{n}=1$ for an experimenter and $k_{n}=0$ for a free-rider, the payoff functions are

$$
\begin{aligned}
u_{n}(p)=\lambda(p) h+(\iota+ & \left.k_{n}-1\right)\left[\frac{r}{r+\lambda_{1}}\left(\lambda_{1} h-s\right) p-\frac{r}{r+\lambda_{0}}\left(s-\lambda_{0} h\right)(1-p)\right] \\
& +C^{(0)}\left(\frac{\lambda_{0}\left(\lambda_{0} / \lambda_{1}\right)^{\mu_{N}}}{\lambda_{0}-\mu_{N} \Delta \lambda}\right)^{\iota} \frac{\lambda_{0}\left(\lambda_{0} / \lambda_{1}\right)^{\mu_{N}}}{r+\lambda_{0}-\mu_{N} \Delta \lambda}(1-p) \Omega(p)^{\mu_{N}} \\
& +\frac{1}{r}\left\{\sum_{\eta=0}^{\iota-1} \frac{C^{(\iota-\eta)}}{\eta !}\left(-\frac{\lambda_{0}\left(\lambda_{0} / \lambda_{1}\right)^{\lambda_{0} / \Delta \lambda}}{\Delta \lambda}\right)^{\eta} \lambda_{0}\left(\lambda_{0} / \lambda_{1}\right)^{\lambda_{0} / \Delta \lambda}\right. \\
& \left.\times\left(\sum_{\gamma=0}^{\eta}\left(\frac{\Delta \lambda}{r}\right)^{\eta-\gamma} \frac{\eta !}{\gamma !}\left(\ln \left[\left(\lambda_{0} / \lambda_{1}\right)^{\gamma} \Omega(p)\right]\right)^{\gamma}\right)\right\}(1-p) \Omega(p)^{\lambda_{0} / \Delta \lambda} \\
& +C_{n}^{(\iota+1)}(1-p) \Omega(p)^{\left(r+\lambda_{0}\right) / \Delta \lambda}
\end{aligned}
$$

for appropriately chosen constants of integration $C_{n}^{(\iota+1)}$; the constants $C^{(i)}, i=0, \ldots, \iota$, are from the common payoff function for beliefs above $p_{N}^{\sharp}$.

With sufficiently frequent turns between the roles of experimenter and free-rider, the players' payoff functions in the equilibria of Proposition 5 become arbitrarily close to the average payoff function. This leads to a Pareto improvement over the symmetric equilibrium.

Proposition 6 (Pareto improvement over the symmetric MPE). The $N$-player experimentation game admits Markov perfect equilibria as in Proposition 5 in which each player's payoff exceeds the symmetric equilibrium payoff on $] p_{N}^{b}, 1[$.

Proof. Let $\delta=(1 / 2) \max _{\tilde{p}_{N} \leq p \leq p_{N}^{\sharp}}\left[\bar{u}(p)-W_{N}(p)\right]$, where $\bar{u}$ is the average payoff function associated with the equilibria of Proposition 5 and $W_{N}$ is the players' common payoff function in the symmetric equilibrium. Choose the subintervals $\left.] p_{\ell, i}, p_{r, i}\right]$ such that $\left|u_{n}-\bar{u}\right|$ is bounded above by $\delta$ for all $n$. Then $u_{n}>s=W_{N}$ on $\left.] p_{N}^{b}, \tilde{p}_{N}\right], u_{n} \geq \bar{u}-\delta>W_{N}$ on $] \tilde{p}_{N}, p_{N}^{\sharp}$, and $u_{n}=\bar{u}>W_{N}$ on $\left.] p_{N}^{\sharp}, 1\right]$.

It deserves to be stressed that it is the encouragement effect that permits Pareto improvements over the symmetric equilibrium. Without it, the last experimenter quits at the same belief (the single-agent cutoff) at which all players stop experimenting in the symmetric equilibrium; bearing all the costs of experimentation on his own, the last experimenter is then necessarily worse off than under symmetry immediately to the right of this cutoff. 
There clearly is scope for further improvements in players' equilibrium payoffs, over and above those embodied in the equilibria of Proposition 5. As we move down from the diagonal $\mathcal{D}_{N-2+1 / N}$ to $\mathcal{D}_{N-2}$, the intensity of experimentation in these equilibria gradually falls from $N-1$ to $N(N-2) /(N-1)$. Using exactly the same approach as on the interval $] p_{N}^{b}, p_{N}^{\sharp}$ ] above, we could instead let players take turns between the roles of experimenter and free-rider such that the intensity of experimentation remains constant at level $N-1$ in between these diagonals.

This raises the question as to whether there exists a best Markov perfect equilibrium, in a sense to be made precise. To shed some light on this question, we focus on the twoplayer case from now on and take the corresponding results for the exponential model as our starting point.

First, the exponential model admits uniformly best and worst two-player equilibria that achieve the maximal (resp. minimal) intensity of experimentation and the maximal (resp. minimal) average payoff compatible with Markov perfection and finite switching, irrespective of the players' initial belief. The worst equilibrium is the symmetric MPE, whereas the best equilibria have the same structure as the two-player equilibria of Proposition 5, with the intensity of experimentation being the limit as $\lambda_{0}$ tends to 0 of the intensity described in that proposition. ${ }^{10}$

Second, the exponential model admits most inequitable equilibria that give the two players extremal individual payoffs. These equilibria involve simple strategies, so that each player uses one arm exclusively at any given belief. ${ }^{11}$ Here there are three thresholds $\bar{p}=p_{1}^{*}<\hat{p}_{s}<\hat{p}_{2}$ such that both players play risky above $\hat{p}_{2}$, one of the players plays risky between $\hat{p}_{s}$ and $\hat{p}_{2}$, the other player plays risky between $\bar{p}$ and $\hat{p}_{s}$, and both play safe below $\bar{p}$. The payoffs achieved by the last experimenter and the last free-rider in these equilibria constitute a uniform lower and upper bound, respectively, on the individual equilibrium payoffs that are compatible with Markov perfection and finite switching.

Our next aim is to investigate whether these most inequitable equilibria can also be obtained as the limit of two-player equilibria of the Poisson model for vanishing $\lambda_{0}>0$. To this end, we first demonstrate the existence of a benchmark simple MPE that gives the players common continuation payoffs after any success on a risky arm. In a numerical example, we then progressively diverge from this benchmark by rewarding the last experimenter, that is, by giving him a higher continuation value after a success than his opponent. This yields equilibria with progressively increasing payoff asymmetry between the players. It also suggests that we cannot expect a uniformly best Markov perfect equilibrium to exist in the Poisson model—not even in the two-player case.

\section{REWARDING THE LAST EXPERIMENTER}

We start with the observation that for $\lambda_{0}$ sufficiently close to zero, any success on a risky arm makes the players so optimistic that playing risky is the dominant action for all of

\footnotetext{
${ }^{10}$ See Proposition 6.3 and footnote 5 of Keller et al. (2005). While the symmetric MPE remains the worst equilibrium of the exponential model for $N>2$, existence and structure of a best MPE are open questions in this case.

${ }^{11}$ See Proposition 6.1 of Keller et al. (2005).
} 
them. More precisely, as any two-player MPE must have an average payoff function in between the single-agent optimum $V_{1}^{*}$ and the cooperative solution $V_{2}^{*}$, the infimum of the set $\{p \mid K(p)>0\}$ must be at least $p_{2}^{*}$. If $j\left(p_{2}^{*}\right) \geq p^{m}$, a success on any risky arm will make players optimistic enough for all of them to revert to exclusive use of the risky arm, and the players' post-jump equilibrium payoffs as well as their average will be of the form $V_{2}(j(p))$ with $V_{2}$ as given in (3).

A necessary and sufficient condition for $j\left(p_{2}^{*}\right) \geq p^{m}$ is that $\mu_{2} /\left(\mu_{2}+1\right) \geq \lambda_{0} / \lambda_{1}$ or, equivalently, that $\mu_{2} \geq \lambda_{0} / \Delta \lambda$. Using (2) with $N=2$, this holds if and only if

$$
\lambda_{0}\left(\frac{\lambda_{0}}{\lambda_{1}}\right)^{\lambda_{0} / \Delta \lambda} \leq \frac{r}{2}
$$

Clearly, since $\lambda_{0} / \lambda_{1}<1$ and $\lambda_{0} / \Delta \lambda>0$, a sufficient condition for (7) is that $\lambda_{0} \leq r / 2$.

When (7) holds, the same approach as in the previous section allows us to construct simple equilibria with symmetric post-jump continuation values. Figure 3 shows the best response correspondence for $N=2$ and illustrates the simplest possible configuration of payoff functions that can arise in the type of equilibrium we construct.

Proposition 7 (Simple MPE for $N=2$ ). Under condition (7), the two-player experimentation game admits simple Markov perfect equilibria with the following features. There are two thresholds, $\bar{p}$ and $\hat{p}$, with $p_{2}^{*}<\bar{p}<\hat{p}<p^{m}$, such that on $\left.] \hat{p}, 1\right]$, both players play $R$ and their payoff functions coincide; on $] \bar{p}, \hat{p}]$, the intensity of experimentation equals 1 , and there is at least one belief in the interior of this interval where both players change action; on $[0, \bar{p}]$, they both play $S$.

We cannot rule out the possibility that the payoff of the last free-rider is nonmonotonic immediately to the right of a switch point (a belief where both players change

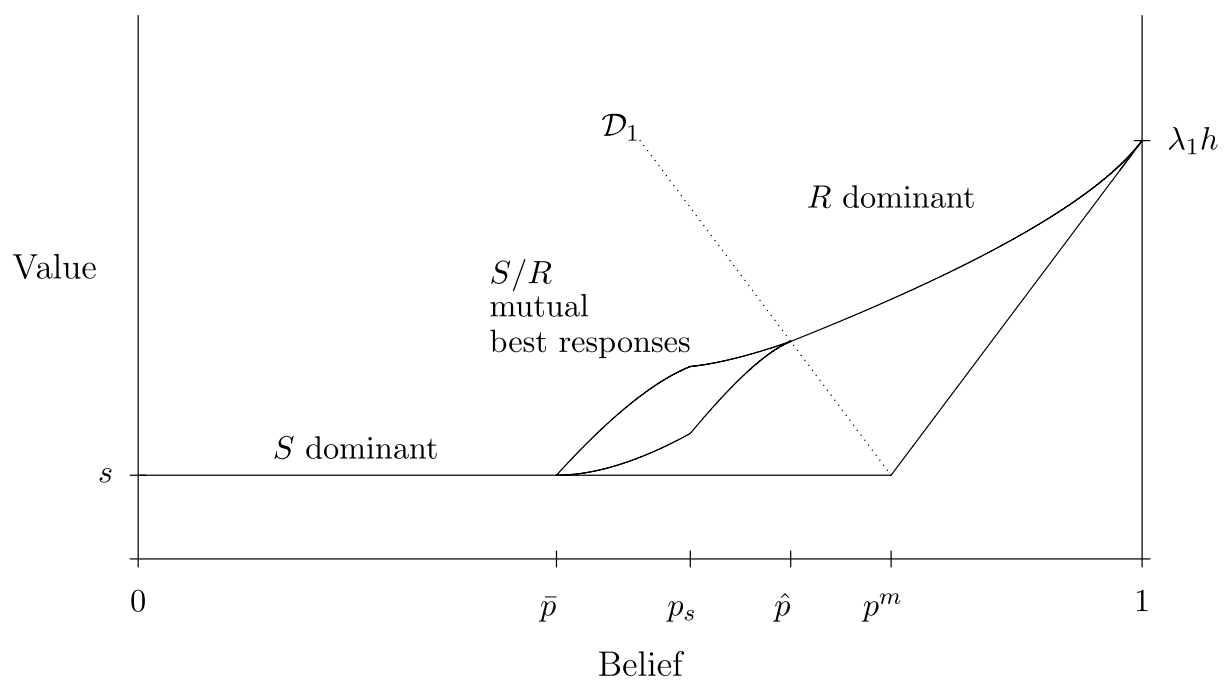

Figure 3. Best responses for $N=2$ and possible payoffs in a simple equilibrium. 
action). ${ }^{12}$ However, Figure 3 illustrates an equilibrium that does have a monotonic payoff function for the last free-rider (the higher of the two payoff functions). This case arises, for example, with the parameter values $r=1, s=1.5, h=2, \lambda_{0}=0.5$, and $\lambda_{1}=1.5$. With these values, the lower threshold $\bar{p}$ is smaller than the belief $\tilde{p}_{2}$ at which all experimentation stops in the symmetric equilibrium, and the average payoff function is greater than the common payoff function in the symmetric equilibrium; this improvement stems again from the fact that the intensity of experimentation remains constant at the level 1 just below $\mathcal{D}_{1 / 2}$, whereas the symmetric equilibrium intensity falls below 1 as soon as $\mathcal{D}_{1 / 2}$ is crossed. ${ }^{13}$

Using condition (7), we can give the following explicit representations for the two players' payoff functions in the equilibria of Proposition 7.

Corollary 4. On ] $\hat{p}, 1]$, where both players experiment, their common payoff function is

$$
u(p)=\lambda(p) h+C^{(0)}(1-p) \Omega(p)^{\mu_{2}},
$$

the constant $C^{(0)}$ being given by

$$
C^{(0)}=2 c(\hat{p})(1-\hat{p})^{-1} \Omega(\hat{p})^{-\mu_{2}} .
$$

On $] \bar{p}, \hat{p}]$, where one player experiments and the other free-rides, and with $k_{n}=1$ for an experimenter and $k_{n}=0$ for a free-rider, the payoff functions are

$$
\begin{aligned}
u_{n}(p)=\lambda(p) h+ & \left(k_{n}-1\right)\left[\frac{r}{r+\lambda_{1}}\left(\lambda_{1} h-s\right) p-\frac{r}{r+\lambda_{0}}\left(s-\lambda_{0} h\right)(1-p)\right] \\
& +C^{(0)}\left(\frac{\lambda_{0}\left(\lambda_{0} / \lambda_{1}\right)^{\mu_{2}}}{r+\lambda_{0}-\mu_{2} \Delta \lambda}\right)(1-p) \Omega(p)^{\mu_{2}}+C_{n}^{(1)}(1-p) \Omega(p)^{\left(r+\lambda_{0}\right) / \Delta \lambda},
\end{aligned}
$$

with appropriately chosen constants of integration $C_{n}^{(1)}$.

Maintaining assumption (7), suppose now that we give the last experimenter a higher payoff at $j(\bar{p})$ than the other player. This has two effects. On the one hand, we can no longer achieve the maximal intensity of $K=2$ immediately to the right of the belief at which the graph of the average payoff function crosses $\mathcal{D}_{1}$, since there the last free-rider's payoff function is necessarily below $\mathcal{D}_{1}$, implying that his best response is to play safe; this lowers average payoffs. On the other hand, the last experimenter is now willing to continue playing $R$ somewhat to the left of $\bar{p}$, which increases average payoffs. We explore this trade-off numerically.

We refer to the last experimenter as player 1 and the last free-rider as player 2 . We continue to let $\bar{p}$ denote the belief where all experimentation stops, and let $p_{s}$ denote the switch point where both players change actions; however, as their payoff functions cross $\mathcal{D}_{1}$ at different points, we let $\hat{p}_{1}$ and $\hat{p}_{2}$ denote the corresponding beliefs.

\footnotetext{
${ }^{12}$ For more on this nonmonotonicity, see the remarks about anticipation near the end of this section.

${ }^{13}$ However, it is not the case that each player is individually better off than in the symmetric MPE; in fact, the last experimenter (the player with the lower of the two payoff functions) is worse off in a neighborhood of the switch point. Subsequently, we present simple equilibria for the above parameter values that are better than the symmetric one for both players.
} 


\section{Construction of equilibria}

The strategies for the players are the following: on ] $\left.\hat{p}_{2}, 1\right]$, both players play $R$; on ] $\hat{p}_{1}, \hat{p}_{2}$ ], player 1 plays $R$ and player 2 plays $S$; on ] $p_{s}, \hat{p}_{1}$ ], player 1 plays $S$ and player 2 plays $R$; on $\left.] \bar{p}, p_{s}\right]$, player 1 plays $R$ and player 2 plays $S$; on $[0, \bar{p}]$, both players play $S{ }^{14}$ We need to determine $\bar{p}<p_{s}<\hat{p}_{1}<\hat{p}_{2}$, and to build continuous functions $u_{1}$ and $u_{2}$ that (a) connect the points $(0, s)$ and $\left(1, \lambda_{1} h\right)$ in the $(p, u)$ plane, that (b) satisfy the appropriate ODDEs, and that (c) have the following properties: $u_{1}$ is above $\mathcal{D}_{1}$ on $\left.] \hat{p}_{1}, 1\right]$, below $\mathcal{D}_{1}$ but above $s$ on $\left.] \bar{p}, \hat{p}_{1}\right]$, at $s$ on $[0, \bar{p}]$, and is smooth at $\bar{p} ; u_{2}$ is above $\mathcal{D}_{1}$ on ]$\left.\hat{p}_{2}, 1\right]$, below $\mathcal{D}_{1}$ but above $s$ on $\left.] \bar{p}, \hat{p}_{2}\right]$, and at $s$ on $[0, \bar{p}]$.

Relative to the upper threshold $\hat{p}$ and the average payoff function $\bar{u}$ from Proposition 7 , choose a point $\left(\hat{p}_{2}, \breve{u}\right)$ in $[0,1] \times\left[s, \lambda_{1} h\right]$ with $\hat{p}_{2}$ to the right of $\hat{p}$ and $\breve{u}$ above $\bar{u}\left(\hat{p}_{2}\right)$. First, we construct player 1's payoff function piecewise.

On ] $\left.\hat{p}_{2}, 1\right]$, both players play $R$, so $u_{1}$ is of the form given in equation (3) with $N=2$, the constant of integration being chosen so that $u_{1}\left(\hat{p}_{2}\right)=\breve{u}$. The belief $\bar{p}$ where player 1 quits can now be determined from equation (1) with $N=1$, using value matching $\left(u_{1}(\bar{p})=s\right)$ and smooth pasting $\left(u_{1}^{\prime}(\bar{p})=0\right)$, and knowing the form of $u_{1}(j(\bar{p}))$ since $j(\bar{p})>\hat{p}_{2}$. Just to the left of $\hat{p}_{2}$, player 1 is the only one playing $R$, so $u_{1}$ is of the form given in Corollary 4 , the constant of integration being chosen to ensure continuity at $\hat{p}_{2}$; $\hat{p}_{1}$ is the belief to the left of $\hat{p}_{2}$ where $u_{1}$ crosses $\mathcal{D}_{1}$. On an interval to the left of that, player 1 is free-riding, $u_{1}$ is again of the form given in Corollary 4 , and the constant of integration is chosen to ensure continuity at $\hat{p}_{1}$. Further, on an interval to the right of $\bar{p}$, player 1 is again the lone experimenter and now the constant of integration is chosen to ensure that $u_{1}(\bar{p})=s$. Player l's switch point is where the graph of $u_{1}$ coming down and to the left from $\left(\hat{p}_{1}, u_{1}\left(\hat{p}_{1}\right)\right)$ intersects the curve going up and to the right from $(\bar{p}, s)$.

Player 2's payoff function is also constructed piecewise. On ] $\left.\hat{p}_{2}, 1\right], u_{2}$ is also of the form given in equation (3) with $N=2$, the constant of integration being chosen so that $u_{2}\left(\hat{p}_{2}\right)$ is on $\mathcal{D}_{1}$. Between $\bar{p}$ and $\hat{p}_{2}, u_{2}$ is of the form given in Corollary 4: player 2 is freeriding on the interval $] \hat{p}_{1}, \hat{p}_{2}$ ] and we ensure continuity at $\hat{p}_{2}$; on an interval to the left of that, player 2 is the lone experimenter and we ensure continuity at $\hat{p}_{1}$; on an interval to the right of $\bar{p}$, player 2 free-rides and the constant of integration is chosen to ensure that $u_{2}(\bar{p})=s$. Player 2's switch point is where the graph of $u_{2}$ coming down and to the left from $\left(\hat{p}_{1}, u_{2}\left(\hat{p}_{1}\right)\right)$ intersects the curve going up and to the right from $(\bar{p}, s)$.

For this to be an equilibrium, we need to have the players switching at the same belief: this involves adjusting $\left(\hat{p}_{2}, \breve{u}\right)$ and iterating until the switch points coincide.

\footnotetext{
${ }^{14}$ Note that this strategy pair requires the players to swap roles once more than in the most inequitable MPE of the exponential model. There, the last experimenter plays risky on the largest possible interval of beliefs $\left.] \bar{p}, \hat{p}_{s}\right]$, which makes his payoff lower than his opponent's at all beliefs in $] \bar{p}, 1[$. This is impossible here. In fact, optimal behavior of player 1 requires $c(\bar{p})=b\left(\bar{p}, u_{1}\right)=\lambda(\bar{p})\left[u_{1}(j(\bar{p}))-s\right] / r$ as the left derivative $u_{1}^{\prime}(\bar{p})=0$. If player 2's payoff were higher than player 1's at $j(\bar{p})$, we would have $b\left(\bar{p}, u_{2}\right)=\lambda(\bar{p})\left[u_{2}(j(\bar{p}))-s\right] / r>c(\bar{p})$, and player 2 would act suboptimally on $[\bar{p}-\epsilon, \bar{p}]$ for some $\epsilon>0$. To reward player 1 , therefore, we need to shorten the interval $] \bar{p}, p_{s}$ ] on which he acts as the last experimenter and let him free-ride to the right of it. This in turn requires a further switch in actions at the belief $\hat{p}_{1}$ where player l's payoff function crosses $\mathcal{D}_{1}$.
} 


\section{Findings}

Using the same parameter values we referred to in the discussion of Figure 3 after Proposition 7 (namely, $r=1, s=1.5, h=2, \lambda_{0}=0.5, \lambda_{1}=1.5$ ), we numerically solved for six equilibria as well as the simple one with common payoffs above $\mathcal{D}_{1}$ (the base case), giving the last experimenter progressively higher payoffs above $\mathcal{D}_{1}$. Figure 4 illustrates the players' payoffs in the base case and in three of these equilibria, the tick labels on the
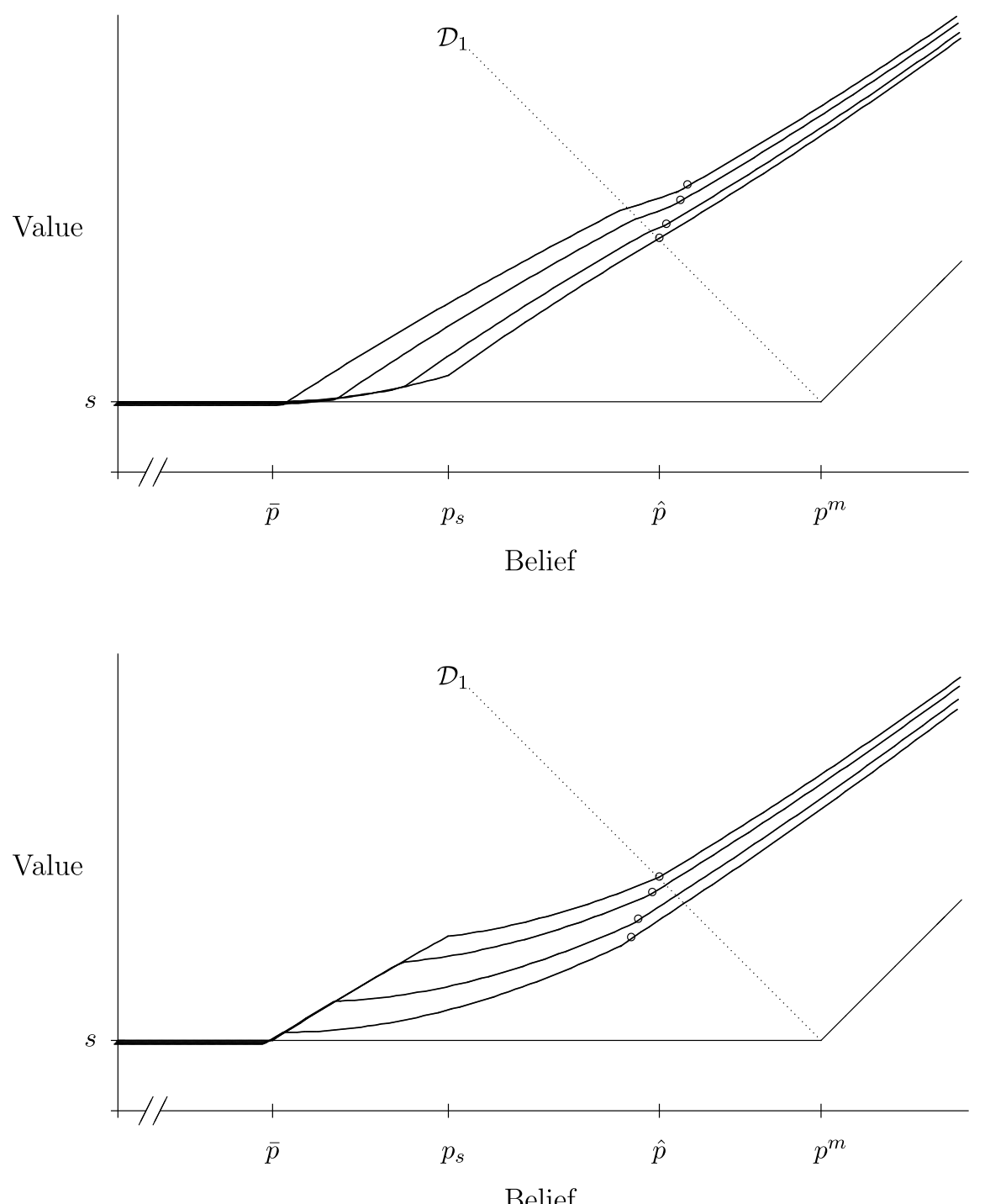

Figure 4. Equilibrium payoffs of the last experimenter (upper panel) and the last free-rider (lower panel). Open circles mark points corresponding to where the other player's value function crosses $\mathcal{D}_{1}$. 
belief axis being for the base case (which exhibits the lowest equilibrium payoffs for the last experimenter and highest for the last free-rider).

We find that as we improve player 1's post-jump payoff, the fall in $\bar{p}$ is about 5 times smaller than the shifts in $\hat{p}_{1}$ and $\hat{p}_{2}$, with $\hat{p}_{1}$ moving to the left and $\hat{p}_{2}$ moving to the right. (The drop in the switch point is more dramatic, being about 25 times that of $\bar{p}$.) The net effect is that the interval of beliefs where exactly one player is experimenting widens, and although the average payoff is higher on an interval to the right of $\bar{p}$, it dips below that of the base case very close to $\hat{p}_{1}$ and remains there at all higher beliefs. Player 1 is progressively better off than in the base case at all beliefs to the right of $\bar{p}$, but player 2 is progressively worse off at beliefs greater than approximately $p_{s}$, and the absolute differences between the average payoff in the base case and those in the other six equilibria become more pronounced as the asymmetry increases. Indeed, in the two most asymmetric of the equilibria that we calculated, player 2's payoff function is below the payoff function in the symmetric equilibrium in a neighborhood of $\hat{p}_{1}$, whereas the payoffs in the other four intermediate equilibria are Pareto improvements on the symmetric equilibrium. Moreover, in the most asymmetric of these equilibria, player 2's payoff function is decreasing in an interval immediately to the right of the switch point (although this is hard to discern visually).

Put another way, in this very asymmetric equilibrium as the players approach the switch point from the right, where player 2 is experimenting alone, beliefs are becoming more pessimistic, yet player 2's payoff is going up. If we put this down to the fact that, conditional on no impending success, player 2 will soon be able to enjoy a free ride, then we can call this an anticipation effect.

More broadly, these findings suggest that by simultaneously letting $p_{s}$ tend to $\bar{p}$ and $\lambda_{0}$ tend to 0 , it is possible to construct a sequence of equilibria that converge to the most inequitable two-player equilibrium of the exponential model. Moreover, as the idea of rewarding the last experimenter can also be applied to the equilibria of Proposition 5, they do not constitute uniformly best equilibria of the Poisson model-not even when $N=2$. By the same token, one is led to conjecture that in marked contrast to the exponential model, the Poisson model does not admit any uniformly best MPE.

\section{Concluding Remarks}

The asymmetric equilibria that we constructed in the Poisson framework raise the question of whether similar equilibria exist in the Brownian model of Bolton and Harris (1999). The elementary constructive method that we used here is likely to apply to the Brownian case as well. Our proof of the result that there exist no equilibria in cutoff strategies should also carry over. We intend to explore this in future work.

Our model can easily be adapted to situations where an event is bad news: a breakdown rather than a breakthrough. For example, we can interpret $s$ as the expected flow cost of keeping the current safe machine running. Players have access to new risky machines that break down and thereby cause lump-sum costs at exponentially distributed times: a high failure rate of $\lambda_{1}$ favors the old machine; a low rate of $\lambda_{0}<\lambda_{1}$ favors the new one. The aim is to minimize the expected sum of discounted costs of breakdowns. 
The longer the machines do not fail, the more optimistic the players become about their reliability, but whenever one does fail, the belief jumps to more pessimistic levels. Given enough pessimism, another failure will be the last straw. Thus, the continuous part of the belief dynamics always keeps the state variable in the continuation region where at least some player uses the new machine, whereas the discontinuous part can cause the state variable to jump into the stopping region. Despite the superficial symmetry between the good-news and the bad-news versions of the model, therefore, the formal analysis of the single-agent optimum, the efficient benchmark, and best responses is rather different from that in the present paper. We defer such analysis to a separate paper.

By constructing simple asymmetric equilibria, our work also prepares the ground for an analysis of strategic experimentation by asymmetric players who might differ, for example, with respect to their innate abilities to achieve breakthroughs, the average size of lump-sum payoffs, or their safe payoffs. This is again left to future work.

\section{ApPENDix}

Proof of Proposition 4 (Details). Let $\hat{p}_{N, N-1}$ denote the belief where the graph of $V_{N}^{*}$ cuts $\mathcal{D}_{N-1}$ and let $\hat{p}_{1, N-1}$ denote the belief where the graph of $V_{1}^{*}$ cuts $\mathcal{D}_{N-1}$. By continuity, there is an open interval $I \supset\left[\hat{p}_{N, N-1}, \hat{p}_{1, N-1}\right]$ such that for all $\hat{p} \in I$, the unique solution to (1) that crosses $\mathcal{D}_{N-1}$ at the belief $\hat{p}$ has positive slope there.

Fix a belief $\hat{p} \in I$ and let $(\hat{p}, \hat{u})$ be the corresponding point on the diagonal $\mathcal{D}_{N-1}$. On $[\hat{p}, 1]$, we define $u^{(0)}$ as the unique solution to (1) that assumes the value $\hat{u}$ at belief $\hat{p}$. Now consider the ordinary differential equation (ODE)

$$
\Delta \lambda p(1-p) u^{\prime}(p)+\lambda(p) u(p)=r \lambda(p) h-r s+\lambda(p) u^{(0)}(j(p)) .
$$

Standard results imply that this ODE has a unique solution $u^{(1)}$ on $\left[j^{-1}(\hat{p}), \hat{p}\right]$ with $u^{(1)}(\hat{p})=u^{(0)}(\hat{p})$ and, by construction, $\left(u^{(1)}\right)^{\prime}(\hat{p})=\left(u^{(0)}\right)^{\prime}(\hat{p})$.

Iterating this step, we construct functions $u^{(i+1)}$ defined on $\left[j^{-(i+1)}(\hat{p}), j^{-i}(\hat{p})\right]$ for $i=1,2,3, \ldots$ by choosing $u^{(i+1)}$ as the unique solution of the ODE

$$
\Delta \lambda p(1-p) u^{\prime}(p)+\lambda(p) u(p)=r \lambda(p) h-r s+\lambda(p) u^{(i)}(j(p))
$$

subject to the condition $u^{(i+1)}\left(j^{-i}(\hat{p})\right)=u^{(i)}\left(j^{-i}(\hat{p})\right)$. Setting $u_{\hat{p}}(p)=u^{(i)}(p)$ whenever $j^{-(i+1)}(\hat{p}) \leq p<j^{-i}(\hat{p})$, we thus obtain a function $u_{\hat{p}}$ of class $C^{1}$ on $\left.] 0,1\right]$ that solves (6) to the left of $\hat{p}$ and solves (1) to the right of $\hat{p}$. Standard results imply that $u_{\hat{p}}$ depends in a continuous fashion on $\hat{p}$. In particular, $M(\hat{p})$, the minimum of $u_{\hat{p}}$ on $\left[p_{N}^{*}, p^{m}\right]$, is continuous in $\hat{p}$.

For $\hat{p} \in I$ with $\hat{p}<\hat{p}_{N, N-1}$, the function $u_{\hat{p}}$ lies above $V_{N}^{*}$ on at least [ $\hat{p}, 1\left[\right.$. If $u_{\hat{p}}$ and $V_{N}^{*}$ assumed the same value at some belief $p_{\ell} \in\left[p_{N}^{*}, \hat{p}\left[\right.\right.$, then the restriction of $u_{\hat{p}}-V_{N}^{*}$ to $\left[p_{\ell}, 1\right]$ would have a positive global maximum at some belief $\left.p_{r} \in\right] p_{\ell}, 1[$. In fact, we would have $\left.p_{r} \in\right] p_{\ell}, \hat{p}$ [, since $u_{\hat{p}}-V_{N}^{*}$, being the difference of two functions of the form (3), has a negative first derivative on $\left[\hat{p}, 1\left[\right.\right.$. As $\left(u_{\hat{p}}\right)^{\prime}\left(p_{r}\right)=\left(V_{N}^{*}\right)^{\prime}\left(p_{r}\right)$ and $u_{\hat{p}}\left(j\left(p_{r}\right)\right)-$ 
$V_{N}^{*}\left(j\left(p_{r}\right)\right) \leq u_{\hat{p}}\left(p_{r}\right)-V_{N}^{*}\left(p_{r}\right)$, we would thus have $b\left(p_{r}, V_{N}^{*}\right) \geq b\left(p_{r}, u_{\hat{p}}\right)=c\left(p_{r}\right)$, hence $V_{N}^{*}\left(p_{r}\right)=s+N b\left(p_{r}, V_{N}^{*}\right)-c\left(p_{r}\right) \geq s+(N-1) c\left(p_{r}\right)$, which is inconsistent with the fact that $V_{N}^{*}$ is below $\mathcal{D}_{N-1}$ at $p_{r}$. Consequently, $u_{\hat{p}}$ lies above $V_{N}^{*}$ on $\left[p_{N}^{*}, 1[\right.$.

By continuity, $\hat{u}_{N}$, the function $u_{\hat{p}}$ obtained for $\hat{p}=\hat{p}_{N, N-1}$, lies weakly above $V_{N}^{*}$ on $\left[p_{N}^{*}, 1\right]$. While the two functions are identical on $\left[\hat{p}_{N, N-1}, 1\right]$ by construction, they cannot be identical on the whole of $\left[p_{N}^{*}, \hat{p}_{N, N-1}\right.$ [ as $V_{N}^{*}$ does not solve (A.1) immediately to the left of $\hat{p}_{N, N-1}$, for example. Arguing exactly as in the previous paragraph, we see that the restriction of $\hat{u}_{N}-V_{N}^{*}$ to $\left[p_{N}^{*}, 1\right]$ must assume its positive global maximum at $p_{N}^{*}$. This establishes $\hat{u}_{N}\left(p_{N}^{*}\right)>V_{N}^{*}\left(p_{N}^{*}\right)=s$. As $V_{N}^{*}(p)>s$ for $p>p_{N}^{*}$, we thus have $\hat{u}_{N}>s$ on $\left[p_{N}^{*}, 1\right]$, hence $M\left(\hat{p}_{N, N-1}\right)>s$.

For $\hat{p} \in I$ with $\hat{p}>\hat{p}_{1, N-1}$, the function $u_{\hat{p}}$ lies below $V_{1}^{*}$ in a neighborhood of $\hat{p}$. If $u_{\hat{p}}$ and $V_{1}^{*}$ assumed the same value at some belief $p_{\ell} \in\left[p_{1}^{*}, \hat{p}[\right.$, then the restriction of $V_{1}^{*}-u_{\hat{p}}$ to $\left[p_{\ell}, 1\right]$ would have a positive global maximum at a belief $\left.p_{r} \in\right] p_{\ell}, 1[$. As $\left(V_{1}^{*}\right)^{\prime}\left(p_{r}\right)=\left(u_{\hat{p}}\right)^{\prime}\left(p_{r}\right)$ and $V_{1}^{*}\left(j\left(p_{r}\right)\right)-u_{\hat{p}}\left(j\left(p_{r}\right)\right) \leq V_{1}^{*}\left(p_{r}\right)-u_{\hat{p}}\left(p_{r}\right)$, we would thus have $b\left(p_{r}, u_{\hat{p}}\right) \geq b\left(p_{r}, V_{1}^{*}\right)$. As $s<V_{1}^{*}\left(p_{r}\right)=s+b\left(p_{r}, V_{1}^{*}\right)-c\left(p_{r}\right)$, this would imply $b\left(p_{r}, u_{\hat{p}}\right)>$ $c\left(p_{r}\right)$ and $p_{r}>\hat{p}$. But then $u_{\hat{p}}\left(p_{r}\right)=s+N b\left(p_{r}, u_{\hat{p}}\right)-c\left(p_{r}\right)>s+b\left(p_{r}, V_{1}^{*}\right)-c\left(p_{r}\right)=$ $V_{1}^{*}\left(p_{r}\right)$, which is a contradiction. Consequently, $u_{\hat{p}}$ lies below $V_{1}^{*}$ on $\left[p_{1}^{*}, \hat{p}\right]$.

By continuity, $\hat{u}_{1, N}$, the function $u_{\hat{p}}$ obtained for $\hat{p}=\hat{p}_{1, N-1}$, lies weakly below $V_{1}^{*}$ on $\left[p_{1}^{*}, \hat{p}_{1, N-1}\right]$. While the two functions are identical at $\hat{p}_{1, N-1}$ by construction, they cannot be identical on the whole of $\left[p_{1}^{*}, \hat{p}_{1, N-1}\right.$ [. Arguing exactly as in the previous paragraph, we see that the restriction of $V_{1}^{*}-\hat{u}_{1, N}$ to $\left[p_{1}^{*}, 1\right]$ must assume its positive global maximum at $p_{1}^{*}$. In particular, $\hat{u}_{1, N}\left(p_{1}^{*}\right)<V_{1}^{*}\left(p_{1}^{*}\right)=s$, hence $M\left(\hat{p}_{1, N-1}\right)<s$.

So there exists a $\left.p_{N}^{\dagger} \in\right] \hat{p}_{N, N-1}, \hat{p}_{1, N-1}$ [ such that $M\left(p_{N}^{\dagger}\right)=s$. With $u^{\dagger}$ denoting the solution $u_{\hat{p}}$ corresponding to $\hat{p}=p_{N}^{\dagger}$, let $\tilde{p}_{N}$ be the highest belief in $\left[p_{N}^{*}, p^{m}\right]$ at which $u^{\dagger}$ assumes the value $s$. By construction, $\tilde{p}_{N}<p_{N}^{\dagger}<p^{m}$. Define the function $W_{N}$ by $W_{N}(p)=s$ on $\left[0, \tilde{p}_{N}\right]$ and by $W_{N}(p)=u^{\dagger}(p)>s$ on $\left.] \tilde{p}_{N}, 1\right]$. This is the common payoff function when all players use the strategy $k$ described in the proposition. As a consequence, $W_{N} \leq V_{N}^{*}$ and, in particular, $\tilde{p}_{N} \geq p_{N}^{*}$.

If we had $\tilde{p}_{N}=p_{N}^{*}$, then $W_{N}\left(p_{N}^{*}\right)=s=V_{N}^{*}\left(p_{N}^{*}\right), W_{N}\left(j\left(p_{N}^{*}\right)\right) \leq V_{N}^{*}\left(j\left(p_{N}^{*}\right)\right)$, and $W_{N}^{\prime}\left(p_{N}^{*}\right)=0=\left(V_{N}^{*}\right)^{\prime}\left(p_{N}^{*}\right)$, implying $b\left(p_{N}^{*}, V_{N}^{*}\right) \geq b\left(p_{N}^{*}, W_{N}\right)$. As $b\left(p_{N}^{*}, V_{N}^{*}\right)=c\left(p_{N}^{*}\right) / N$, $b\left(p_{N}^{*}, W_{N}\right)=c\left(p_{N}^{*}\right)$, and $c\left(p_{N}^{*}\right)>0$, this is a contradiction. So we have $p_{N}^{*}<\tilde{p}_{N}<p^{m}$, hence $W_{N}^{\prime}\left(\tilde{p}_{N}+\right)=\left(u^{\dagger}\right)^{\prime}\left(\tilde{p}_{N}\right)=0$ because the minimum of $u^{\dagger}$ on $\left[p_{N}^{*}, p^{m}\right]$ is achieved at an interior point. Thus, the function $W_{N}$ is of class $C^{1}$.

It is straightforward to check from the explicit representation of $W_{N}$ above $\mathcal{D}_{N-1}$ that this function is convex and increasing on $\left[p_{N}^{\dagger}, 1\right]$. Suppose $W_{N}$ is not increasing on $\left[\tilde{p}_{N}, p_{N}^{\dagger}\right]$. Then it must assume both a local maximum and a local minimum in the interior of that interval, and there exist beliefs $p^{\prime}<p^{\prime \prime}$ in $] \tilde{p}_{N}, p_{N}^{\dagger}\left[\right.$ such that $W_{N}^{\prime}\left(p^{\prime}\right)=$ $W_{N}^{\prime}\left(p^{\prime \prime}\right)=0, W_{N}\left(p^{\prime}\right) \geq W_{N}\left(p^{\prime \prime}\right)$, and $W_{N}$ is weakly decreasing on $\left[p^{\prime}, p^{\prime \prime}\right]$ and increasing on $\left[p^{\prime \prime}, 1\right]$. We now have $b\left(p^{\prime}, W_{N}\right)=\lambda\left(p^{\prime}\right)\left[W_{N}\left(j\left(p^{\prime}\right)\right)-W_{N}\left(p^{\prime}\right)\right] / r=c\left(p^{\prime}\right)>0$, hence $W_{N}\left(j\left(p^{\prime}\right)\right)>W_{N}\left(p^{\prime}\right)$ and $j\left(p^{\prime}\right)>p^{\prime \prime}$. As a consequence, $W_{N}\left(j\left(p^{\prime \prime}\right)\right)>W_{N}\left(j\left(p^{\prime}\right)\right)$ and $b\left(p^{\prime \prime}, W_{N}\right)=\lambda\left(p^{\prime \prime}\right)\left[W_{N}\left(j\left(p^{\prime \prime}\right)\right)-W_{N}\left(p^{\prime \prime}\right)\right] / r>\lambda\left(p^{\prime}\right)\left[W_{N}\left(j\left(p^{\prime}\right)\right)-W_{N}\left(p^{\prime}\right)\right] / r=c\left(p^{\prime}\right)>$ $c\left(p^{\prime \prime}\right)$, which is a contradiction. This establishes that $W_{N}$ is increasing on $\left[\tilde{p}_{N}, 1\right]$, and $k$ is increasing on $\left[\tilde{p}_{N}, p_{N}^{\dagger}\right]$. 
We thus have $b\left(p, W_{N}\right)>c(p)$ on $\left.] p_{N}^{\dagger}, 1\right], b\left(p, W_{N}\right)=c(p)$ on $\left[\tilde{p}_{N}, p_{N}^{\dagger}\right]$ and, because of the monotonicity of $W_{N}$ on $\left[\tilde{p}_{N}, 1\right], b\left(p, W_{N}\right)<c(p)$ on $\left[0, \tilde{p}_{N}\right.$ [. So all players using the strategy $k$ constitutes an equilibrium. Finally, $\tilde{p}_{N}<p_{1}^{*}$ by Proposition 2.

Uniqueness was already shown in the main text.

Proof of Corollary 1. With $u^{(0)}(p)=\lambda_{1} h p+\lambda_{0} h(1-p)+C^{(0)}(1-p) \Omega(p)^{\mu}$ (see (3)), we seek a sequence of functions $u^{(i+1)}$ for $i=0,1, \ldots$, defined recursively as solutions to the ODE (A.2). Let $\alpha=\lambda_{0} / \Delta \lambda$ and, for $i \geq 0$, let

$$
\begin{aligned}
u^{(i)}(p)=d_{1}^{(i)} p+d_{0}^{(i)}(1-p)+m^{(i)} & (1-p) \Omega(p)^{\mu} \\
& +(1-p) \Omega(p)^{\alpha} \sum_{\eta=0}^{i-1} l^{(i-\eta)}\left(\ln \left[\left(\lambda_{0} / \lambda_{1}\right)^{\eta-1} \Omega(p)\right]\right)^{\eta},
\end{aligned}
$$

where $d_{1}^{(i)}, d_{0}^{(i)}, m^{(i)}$, and $l^{(i-\eta)}$ are constants to be determined-we will show that the functions $u^{(i)}$ form just such a sequence. Clearly we need

$$
d_{1}^{(0)}=\lambda_{1} h, \quad d_{0}^{(0)}=\lambda_{0} h, \quad \text { and } \quad m^{(0)}=C^{(0)}
$$

with $C^{(0)}$ being the constant that fixes payoffs above the diagonal where everyone plays $R$. The final (summed) term in the above equation defining $u^{(i)}$ is vacuous for $i=0$.

First note that

$$
\begin{aligned}
u^{(i)}(j(p))=d_{1}^{(i)} \frac{\lambda_{1}}{\lambda(p)} p+ & d_{0}^{(i)} \frac{\lambda_{0}}{\lambda(p)}(1-p)+m^{(i)} \frac{\lambda_{0}}{\lambda(p)}\left(\frac{\lambda_{0}}{\lambda_{1}}\right)^{\mu}(1-p) \Omega(p)^{\mu} \\
& +\frac{\lambda_{0}}{\lambda(p)}\left(\frac{\lambda_{0}}{\lambda_{1}}\right)^{\alpha}(1-p) \Omega(p)^{\alpha} \sum_{\eta=0}^{i-1} l^{(i-\eta)}\left(\ln \left[\left(\lambda_{0} / \lambda_{1}\right)^{\eta} \Omega(p)\right]\right)^{\eta},
\end{aligned}
$$

so that the right-hand side of (A.2) becomes

$$
\begin{aligned}
G^{(i)}(p)=D_{1}^{(i)} p+D_{0}^{(i)}(1-p)+M^{(i)} & (1-p) \Omega(p)^{\mu} \\
& +(1-p) \Omega(p)^{\alpha} \sum_{\eta=0}^{i-1} L^{(i-\eta)}\left(\ln \left[\left(\lambda_{0} / \lambda_{1}\right)^{\eta} \Omega(p)\right]\right)^{\eta},
\end{aligned}
$$

where

$$
D_{1}^{(i)}=d_{1}^{(i)} \lambda_{1}+r\left(\lambda_{1} h-s\right), \quad D_{0}^{(i)}=d_{0}^{(i)} \lambda_{0}-r\left(s-\lambda_{0} h\right)
$$

and

$$
M^{(i)}=m^{(i)} \lambda_{0}\left(\lambda_{0} / \lambda_{1}\right)^{\mu}, \quad L^{(i-\eta)}=l^{(i-\eta)} \lambda_{0}\left(\lambda_{0} / \lambda_{1}\right)^{\alpha} .
$$

The homogeneous equation, $\Delta \lambda p(1-p) u^{\prime}(p)+\lambda(p) u(p)=0$, has the solution

$$
u_{0}(p)=(1-p) \Omega(p)^{\alpha} .
$$


Using the method of variation of constants, we now write $u(p)=a(p) u_{0}(p)$ so that

$$
\Delta \lambda p(1-p) u^{\prime}(p)+\lambda(p) u(p)=\Delta \lambda p(1-p) u_{0}(p) a^{\prime}(p) .
$$

The ODE thus transforms into the following equation for the first derivative of the unknown function $a$ :

$$
\begin{aligned}
\Delta \lambda a^{\prime}(p)= & \frac{G^{(i)}(p)}{p(1-p) u_{0}(p)} \\
= & D_{1}^{(i)} \Omega(p)^{-\alpha}(1-p)^{-2}+ \\
& D_{0}^{(i)} \Omega(p)^{-\alpha+1}(1-p)^{-2}+M^{(i)} \Omega(p)^{\mu-\alpha+1}(1-p)^{-2} \\
& +\Omega(p)(1-p)^{-2} \sum_{\eta=0}^{i-1} L^{(i-\eta)}\left(\ln \left[\left(\lambda_{0} / \lambda_{1}\right)^{\eta} \Omega(p)\right]\right)^{\eta} .
\end{aligned}
$$

Make the substitution $\omega=\Omega(p)$ and define $A(\omega)=a(p)$, so $a^{\prime}(p)=-A^{\prime}(\omega) / p^{2}$. Then

$$
-\Delta \lambda A^{\prime}(\omega)=D_{1}^{(i)} \omega^{-\alpha-2}+D_{0}^{(i)} \omega^{-\alpha-1}+M^{(i)} \omega^{\mu-\alpha-1}+\omega^{-1} \sum_{\eta=0}^{i-1} L^{(i-\eta)}\left(\ln \left[\left(\lambda_{0} / \lambda_{1}\right)^{\eta} \omega\right]\right)^{\eta},
$$

so

$$
\begin{aligned}
& A(\omega)=\frac{D_{1}^{(i)}}{\lambda_{1}} \omega^{-\alpha-1}+\frac{D_{0}^{(i)}}{\lambda_{0}} \omega^{-\alpha}+\frac{M^{(i)}}{\lambda_{0}-\mu \Delta \lambda} \omega^{\mu-\alpha} \\
& \quad-\sum_{\eta=0}^{i-1} \frac{L^{(i-\eta)}}{(\eta+1) \Delta \lambda}\left(\ln \left[\left(\lambda_{0} / \lambda_{1}\right)^{\eta} \omega\right]\right)^{\eta+1}+C^{(i+1)},
\end{aligned}
$$

where $C^{(i+1)}$ is a constant of integration (and assuming $\mu \neq \alpha$, else we have another logarithmic term). Multiplying by $u_{0}(p)=(1-p) \omega^{\alpha}$ and substituting $\omega=\Omega(p)$ leads to

$$
\begin{aligned}
u^{(i+1)}(p)= & \frac{D_{1}^{(i)}}{\lambda_{1}} p+\frac{D_{0}^{(i)}}{\lambda_{0}}(1-p)+\frac{M^{(i)}}{\lambda_{0}-\mu \Delta \lambda}(1-p) \Omega(p)^{\mu} \\
& +(1-p) \Omega(p)^{\alpha} \sum_{\eta=1}^{i} \frac{-L^{(i+1-\eta)}}{\eta \Delta \lambda}\left(\ln \left[\left(\lambda_{0} / \lambda_{1}\right)^{\eta-1} \Omega(p)\right]\right)^{\eta}+(1-p) \Omega(p)^{\alpha} C^{(i+1)} .
\end{aligned}
$$

The above iterative step shows that

$$
d_{1}^{(i+1)}=d_{1}^{(i)}+\frac{r}{\lambda_{1}}\left(\lambda_{1} h-s\right), \quad d_{0}^{(i+1)}=d_{0}^{(i)}-\frac{r}{\lambda_{0}}\left(s-\lambda_{0} h\right),
$$

and

$$
m^{(i+1)}=m^{(i)}\left(\frac{\lambda_{0}\left(\lambda_{0} / \lambda_{1}\right)^{\mu}}{\lambda_{0}-\mu \Delta \lambda}\right)
$$

and so, in general,

$$
d_{1}^{(i)}=\lambda_{1} h+\frac{r}{\lambda_{1}}\left(\lambda_{1} h-s\right) i, \quad d_{0}^{(i)}=\lambda_{0} h-\frac{r}{\lambda_{0}}\left(s-\lambda_{0} h\right) i,
$$


and

$$
m^{(i)}=C^{(0)}\left(\frac{\lambda_{0}\left(\lambda_{0} / \lambda_{1}\right)^{\mu}}{\lambda_{0}-\mu \Delta \lambda}\right)^{i}
$$

After a little algebra, we find that the constants in the summation are given by

$$
l^{(i-\eta)}=\frac{C^{(i-\eta)}}{\eta !}\left(-\frac{\lambda_{0}\left(\lambda_{0} / \lambda_{1}\right)^{\alpha}}{\Delta \lambda}\right)^{\eta} \text { for } \eta=0, \ldots, i-1 .
$$

The constants $C^{(i-\eta)}(\eta=0, \ldots, i-1)$ are chosen to ensure continuity. In particular, writing $\hat{j}^{-i}$ for $j^{-i}\left(p_{N}^{\dagger}\right), C^{(i+1)}$ is chosen such that $u^{(i+1)}\left(\hat{\jmath}^{-i}\right)=u^{(i)}\left(\hat{\jmath}^{-i}\right)$ for $i \geq 0$ and satisfies

$$
\begin{aligned}
C^{(i+1)}\left(1-\hat{\jmath}^{-i}\right) \Omega\left(\hat{\jmath}^{-i}\right)^{\alpha}=- & \frac{r}{\lambda_{1}}\left(\lambda_{1} h-s\right) \hat{\jmath}^{-i}+\frac{r}{\lambda_{0}}\left(s-\lambda_{0} h\right)\left(1-\hat{\jmath}^{-i}\right) \\
& +C^{(0)}\left(1-\frac{\lambda_{0}\left(\lambda_{0} / \lambda_{1}\right)^{\mu}}{\lambda_{0}-\mu \Delta \lambda}\right)\left(\frac{\lambda_{0}\left(\lambda_{0} / \lambda_{1}\right)^{\mu}}{\lambda_{0}-\mu \Delta \lambda}\right)^{i}\left(1-\hat{\jmath}^{-i}\right) \Omega\left(\hat{\jmath}^{-i}\right)^{\mu} \\
+ & \left\{\sum _ { \eta = 0 } ^ { i - 1 } C ^ { ( i - \eta ) } \left[\frac{1}{\eta !}\left(-\frac{\lambda_{0}\left(\lambda_{0} / \lambda_{1}\right)^{\alpha}}{\Delta \lambda} \ln \left[\left(\lambda_{0} / \lambda_{1}\right)^{\eta-1} \Omega\left(\hat{\jmath}^{-i}\right)\right]\right)^{\eta}\right.\right. \\
& \left.\left.-\frac{1}{(\eta+1) !}\left(-\frac{\lambda_{0}\left(\lambda_{0} / \lambda_{1}\right)^{\alpha}}{\Delta \lambda} \ln \left[\left(\lambda_{0} / \lambda_{1}\right)^{\eta} \Omega\left(\hat{\jmath}^{-i}\right)\right]\right)^{\eta+1}\right]\right\} \\
& \times\left(1-\hat{\jmath}^{-i}\right) \Omega\left(\hat{\jmath}^{-i}\right)^{\alpha} .
\end{aligned}
$$

Proof of Corollary 2. Close to the right of $\tilde{p}_{N}$, the dynamics of the belief $p$ given no success are

$$
d p=-\Delta \lambda \frac{N}{N-1} \frac{W_{N}(p)-s}{c(p)} p(1-p) d t .
$$

(A success merely causes a delay before the belief decays to near $\tilde{p}_{N}$ again.) As $W_{N}$ is of class $C^{2}$ to the right of $\tilde{p}_{N}$ with $W_{N}\left(\tilde{p}_{N}\right)=s, W_{N}^{\prime}\left(\tilde{p}_{N}\right)=0$, and $W_{N}^{\prime \prime}\left(\tilde{p}_{N}+\right) \geq 0$, we can find a positive constant $C$ such that

$$
\Delta \lambda \frac{N}{N-1} \frac{W_{N}(p)-s}{c(p)} p(1-p)<C\left(p-\tilde{p}_{N}\right)^{2}
$$

in a neighborhood of $\tilde{p}_{N}$. Starting from an initial belief $p_{0}>\tilde{p}_{N}$ in this neighborhood, consider the dynamics $d p=-C\left(p-\tilde{p}_{N}\right)^{2} d t$. The solution with initial value $p_{0}$,

$$
p_{t}=\tilde{p}_{N}+\frac{1}{C t+\left(p_{0}-\tilde{p}_{N}\right)^{-1}},
$$

does not reach $\tilde{p}_{N}$ in finite time. Since the modified dynamics decrease faster than the original, this result carries over to the true evolution of beliefs.

Proof of Proposition 5 (Details). Let $\hat{p}_{N, N-1}$ denote the belief where the graph of $V_{N}^{*}$ cuts $\mathcal{D}_{N-1}$, and consider $I=\left[\hat{p}_{N, N-1}-\epsilon, p^{m}\right]$ with $\epsilon>0$ small enough that for all $\hat{p} \in I$, the unique solution to (1) that crosses $\mathcal{D}_{N-1}$ at the belief $\hat{p}$ has positive slope there. 
Step 1: Construction of the average payoff function. Fix a belief $\hat{p} \in I$. On $[\hat{p}, 1]$, we define $u_{\hat{p}}$ as the unique solution to (1) that starts on $\mathcal{D}_{N-1}$ at $\hat{p}$. Starting from this initial condition, we then proceed iteratively as in the proof of Proposition 4 , solving "forward" towards lower beliefs and eventually to $p_{N}^{*}$. Between $\mathcal{D}_{N-1}$ and $\overline{\mathcal{D}} \wedge \mathcal{D}_{1-1 / N}$, we solve the indifference ODDE (6); below $\overline{\mathcal{D}} \wedge \mathcal{D}_{1-1 / N}$, we solve the ODDE

$$
\Delta \lambda p(1-p) u^{\prime}(p)=\lambda(p)[u(j(p))-u(p)]-r[u(p)-s]+\frac{r}{N}[\lambda(p) h-s] .
$$

In this manner, we obtain a continuous function $u_{\hat{p}}$ on $\left[p_{N}^{*}, 1\right]$ such that (i) $u_{\hat{p}}(p)=$ $s+N b\left(p, u_{\hat{p}}\right)-c(p)$ and $b\left(p, u_{\hat{p}}\right)>c(p)$ on $\left.] \hat{p}, 1\right]$; (ii) $b\left(p, u_{\hat{p}}\right)=c(p)$ at all beliefs $\left.p \in] p_{N}^{*}, \hat{p}\right]$ where the point $\left(p, u_{\hat{p}}(p)\right)$ lies on or below $\mathcal{D}_{N-1}$ and above $\overline{\mathcal{D}} \wedge \mathcal{D}_{1-1 / N}$; (iii) $u_{\hat{p}}(p)=s+b\left(p, u_{\hat{p}}\right)-c(p) / N$ and $b\left(p, u_{\hat{p}}\right) \leq c(p)$ at all beliefs $\left.p \in\right] p_{N}^{*}, \hat{p}$ ] where $\left(p, u_{\hat{p}}(p)\right)$ lies on or below $\overline{\mathcal{D}} \wedge \mathcal{D}_{1-1 / N}$.

Again proceeding as in the proof of Proposition 4, we establish the existence of a $\hat{p} \in] \hat{p}_{N, N-1}, p^{m}$ [ such that the corresponding function $u_{\hat{p}}$ has an interior global minimum equal to $s$ at some belief $\breve{p} \in] p_{N}^{*}, \hat{p}$. As $u_{\hat{p}}^{\prime}(\breve{p})=0$, we have $\lambda(\breve{p})\left[u_{\hat{p}}(j(\breve{p}))-\right.$ $s] / r=b\left(\breve{p}, u_{\hat{p}}\right)=c(\breve{p}) / N<c(\breve{p})$. For $\hat{p}=p^{m}$, alternatively, the corresponding function $u_{\hat{p}}$ assumes value $s$ at $p^{m}$. As its slope there is positive and $c\left(p^{m}\right)=0$, we have $\lambda\left(p^{m}\right)\left[u_{\hat{p}}\left(j\left(p^{m}\right)\right)-s\right] / r>b\left(p^{m}, u_{\hat{p}}\right)=c\left(p^{m}\right) / N=c\left(p^{m}\right)$. By continuity of $u_{\hat{p}}$ with respect to $\hat{p}$, there exists $\left.p_{N}^{\ddagger} \in\right] \hat{p}_{N, N-1}, p^{m}$ [ such that $u_{p_{N}^{\ddagger}}$, the function $u_{\hat{p}}$ obtained for $\hat{p}=p_{N}^{\ddagger}$, has the following property: there is a belief $\left.p_{N}^{b} \in\right] \breve{p}, p_{N}^{\ddagger}\left[\right.$ such that $u_{p_{N}^{\ddagger}}\left(p_{N}^{\mathrm{b}}\right)=s$, $u_{p_{N}^{\ddagger}}(p)>s$ for $p>p_{N}^{b}$, and $\lambda\left(p_{N}^{b}\right)\left[u_{p_{N}^{\ddagger}}\left(j\left(p_{N}^{b}\right)\right)-s\right] / r=c\left(p_{N}^{b}\right)$.

We define a function $\bar{u}$ on $[0,1]$ by taking $\bar{u}=u_{p_{N}^{\ddagger}}$ on $\left[p_{N}^{\mathrm{b}}, 1\right]$ and $\bar{u}=s$ everywhere else. We want to establish that $\bar{u}$ is increasing on $\left[p_{N}^{b}, 1\right]$. The explicit representation (3) makes this obvious on $\left[p_{N}^{\ddagger}, 1\right]$. Moreover, the argument given in the proof of Proposition 4 shows that $\bar{u}$ is also increasing on $\left[p_{N}^{\sharp}, p_{N}^{\ddagger}\right.$, where $p_{N}^{\sharp}$ is the rightmost belief at which the graph of $\bar{u}$ crosses $\overline{\mathcal{D}} \wedge \mathcal{D}_{1-1 / N}$. Suppose now that $\bar{u}$ is not increasing on $\left[p_{N}^{b}, p_{N}^{\sharp}\right]$. Then there exist beliefs $p^{\prime}<p^{\prime \prime}$ in $\left.] p_{N}^{b}, p_{N}^{\sharp}\right]$ such that $\bar{u}^{\prime}\left(p^{\prime}-\right) \geq 0$, $\bar{u}^{\prime}\left(p^{\prime \prime}-\right) \leq 0$, and $\bar{u}$ is weakly decreasing on $\left[p^{\prime}, p^{\prime \prime}\right]$. As $j\left(p^{\prime \prime}\right)>j\left(p^{\prime}\right)>j\left(p_{N}^{b}\right) \geq p_{N}^{\sharp}$, we have $\bar{u}\left(j\left(p^{\prime \prime}\right)\right)>\bar{u}\left(j\left(p^{\prime}\right)\right)$, hence $\bar{u}\left(j\left(p^{\prime \prime}\right)\right)-\bar{u}\left(p^{\prime \prime}\right)>\bar{u}\left(j\left(p^{\prime}\right)\right)-\bar{u}\left(p^{\prime}\right)$ and $b\left(p^{\prime \prime}, \bar{u}\right)>$ $b\left(p^{\prime}, \bar{u}\right)$. This implies $\bar{u}\left(p^{\prime}\right)=s+b\left(p^{\prime}, \bar{u}\right)-c\left(p^{\prime}\right) / N<s+b\left(p^{\prime \prime}, \bar{u}\right)-c\left(p^{\prime \prime}\right) / N=\bar{u}\left(p^{\prime \prime}\right)-\mathrm{a}$ contradiction.

Monotonicity immediately implies that $\bar{u}$ is the average payoff function associated with the intensity of experimentation: $K(p)=N$ for $p \geq p_{N}^{\ddagger} ; K(p)=N \bar{u}(p) /$ $[(N-1) c(p)]<N$ for $p_{N}^{\sharp}<p<p_{N}^{\ddagger} ; K(p)=1$ for $p_{N}^{b}<p \leq p_{N}^{\sharp} ; K(p)=0$ for $p \leq p_{N}^{b}$. Using the explicit form of the relevant ODDE to the left and right of $p_{N}^{\sharp}$, respectively, we see that $\Delta \lambda p_{N}^{\sharp}\left(1-p_{N}^{\sharp}\right)\left[\bar{u}^{\prime}\left(p_{N}^{\sharp}+\right)-\bar{u}^{\prime}\left(p_{N}^{\sharp}-\right)\right]=r\left[\bar{u}\left(p_{N}^{\sharp}\right)-s-(1-1 / N) c\left(p_{N}^{\sharp}\right)\right]$, so $\bar{u}$ has a kink at $p_{N}^{\sharp}$ with $\bar{u}^{\prime}\left(p_{N}^{\sharp}-\right)>\bar{u}^{\prime}\left(p_{N}^{\sharp}+\right)$ if and only if the intersection with $\overline{\mathcal{D}} \wedge \mathcal{D}_{1-1 / N}$ is below $\mathcal{D}_{1-1 / N}$. This kink then corresponds to a jump in the intensity of experimentation with $K\left(p_{N}^{\sharp}-\right)=1>K\left(p_{N}^{\sharp}+\right)$. By construction, $K$ always jumps at $p_{N}^{b}$ and $\bar{u}$ always has a kink there. At all other beliefs, $K$ is continuous and $\bar{u}$ is once continuously differentiable.

Step 2: Construction of the players' payoff functions and strategies. We define

$$
\bar{b}(p, u)=\left[\lambda(p)(\bar{u}(j(p))-u(p))-\Delta \lambda p(1-p) u^{\prime}(p)\right] / r
$$


for any left-differentiable real-valued function $u$ on $] 0,1]$. (This is the benefit of experimentation when the value after a success is given by the payoff function $\bar{u}$.)

Fix any two beliefs $p_{\ell}<p_{r}$ in $\left[p_{N}^{\mathrm{b}}, p_{N}^{\sharp}\right]$ and consider the four functions $u_{\ell F}, u_{\ell E}$, $u_{r F}$, and $u_{r E}$ on $\left[p_{\ell}, p_{r}\right]$ that are uniquely determined by the properties $u_{\ell F}\left(p_{\ell}\right)=$ $u_{\ell E}\left(p_{\ell}\right)=\bar{u}\left(p_{\ell}\right) ; u_{r F}\left(p_{r}\right)=u_{r E}\left(p_{r}\right)=\bar{u}\left(p_{r}\right) ;$ on $\left.] p_{\ell}, p_{r}\right], u_{\ell F}$ and $u_{r F}$ solve the freerider ODE $u(p)=s+\bar{b}(p, u)$, while $u_{\ell E}$ and $u_{r E}$ solve the experimenter ODE $u(p)=$ $s+\bar{b}(p, u)-c(p)$. By construction, $\left[(N-1) u_{\ell F}+u_{\ell E}\right] / N$ coincides with $\bar{u}$ at $p_{\ell}$ and solves the same ODE as $\bar{u}$ on $] p_{\ell}, p_{r}$ ], namely $u(p)=s+\bar{b}(p, u)-c(p) / N$, so it must coincide with $\bar{u}$ on $\left[p_{\ell}, p_{r}\right]$. The same argument applies to $\left[(N-1) u_{r F}+u_{r E}\right] / N$. We can thus conclude that $(N-1) u_{\ell F}+u_{\ell E}=(N-1) u_{r F}+u_{r E}$ on $\left[p_{\ell}, p_{r}\right]$.

Next, we have $u_{\ell F}^{\prime}\left(p_{\ell}+\right)>\bar{u}^{\prime}\left(p_{\ell}+\right)$ since $\lim _{p \downarrow p_{\ell}} \bar{b}\left(p, u_{\ell F}\right)=\bar{b}\left(p_{\ell}, \bar{u}\right)-c\left(p_{\ell}\right) / N<$ $\bar{b}\left(p_{\ell}, \bar{u}\right)$ and $\lim _{p \downarrow p_{\ell}}\left[\bar{u}(j(p))-u_{\ell F}(p)\right]=\bar{u}\left(j\left(p_{\ell}\right)\right)-\bar{u}\left(p_{\ell}\right)$. Thus, $u_{\ell F}(p)>\bar{u}(p)$ immediately to the right of $p_{\ell}$. Now there cannot exist a belief $\left.p^{\prime} \in\right] p_{\ell}, p_{r}$ ] such that $u_{\ell F}\left(p^{\prime}\right)=\bar{u}\left(p^{\prime}\right)$ and $u_{\ell F}^{\prime}\left(p^{\prime}\right) \leq \bar{u}^{\prime}\left(p^{\prime}\right)$, because we would then have $c\left(p^{\prime}\right) / N=b\left(p^{\prime}, \bar{u}\right)-$ $\bar{b}\left(p^{\prime}, u_{\ell F}\right)=-\Delta \lambda p^{\prime}\left(1-p^{\prime}\right)\left[\bar{u}^{\prime}\left(p^{\prime}\right)-u_{\ell F}^{\prime}\left(p^{\prime}\right)\right] / r \leq 0-$ a contradiction. This implies that $u_{\ell F}>\bar{u}$ on the entire interval $\left.] p_{\ell}, p_{r}\right]$. Analogous arguments establish that $u_{\ell E}<\bar{u}$ on ]$\left.p_{\ell}, p_{r}\right]$ as well as $u_{r F}<\bar{u}$ and $u_{r E}>\bar{u}$ on $\left[p_{\ell}, p_{r}[\right.$.

In particular, there exists a belief $p \in] p_{\ell}, p_{r}$ [ such that $u_{\ell E}(p)=u_{r F}(p)$. Let $p_{1}$ denote the lowest such belief and define a continuous function $u_{1}\left(\cdot \mid p_{\ell}, p_{r}\right)$ on $\left[p_{\ell}, p_{r}\right]$ by setting $u_{1}\left(\cdot \mid p_{\ell}, p_{r}\right)=u_{\ell E}$ on $\left[p_{\ell}, p_{1}\right]$ and $u_{1}\left(\cdot \mid p_{\ell}, p_{r}\right)=u_{r F}$ on $\left[p_{1}, p_{r}\right]$. Using the identity $(N-1) u_{\ell F}+u_{\ell E}=(N-1) u_{r F}+u_{r E}$, we see that $u_{r E}\left(p_{1}\right)-u_{\ell F}\left(p_{1}\right)=(N-2)\left[u_{\ell F}\left(p_{1}\right)-\right.$ $\left.u_{r F}\left(p_{1}\right)\right]$. If $N=2$, we define a continuous function $u_{2}\left(\cdot \mid p_{\ell}, p_{r}\right)$ on $\left[p_{\ell}, p_{r}\right]$ by setting $u_{2}\left(\cdot \mid p_{\ell}, p_{r}\right)=u_{\ell F}$ on $\left[p_{\ell}, p_{1}\right]$ and $u_{2}\left(\cdot \mid p_{\ell}, p_{r}\right)=u_{r E}$ on $\left[p_{1}, p_{r}\right]$.

If $N>2$, we consider the function $u_{[2]}$ that coincides with $u_{\ell F}$ at $p_{1}$ and solves the experimenter ODE $u(p)=s+\bar{b}(p, u)-c(p)$ on $\left.] p_{1}, p_{r}\right]$. As $u_{r E}\left(p_{1}\right)>u_{\ell F}\left(p_{1}\right)$, there is a belief $p \in] p_{1}, p_{r}$ [ such that $u_{[2]}(p)=u_{r F}(p)$. Let $p_{2}$ denote the lowest such belief and define a continuous function $u_{2}\left(\cdot \mid p_{\ell}, p_{r}\right)$ on $\left[p_{\ell}, p_{r}\right]$ by setting $u_{2}\left(\cdot \mid p_{\ell}, p_{r}\right)=u_{\ell F}$ on $\left[p_{\ell}, p_{1}\right], u_{2}\left(\cdot \mid p_{\ell}, p_{r}\right)=u_{[2]}$ on $\left[p_{1}, p_{2}\right]$, and $u_{2}\left(\cdot \mid p_{\ell}, p_{r}\right)=u_{r F}$ on $\left[p_{2}, p_{r}\right]$. By the same argument as above, $(N-2) u_{\ell F}+u_{[2]}+u_{r F}=(N-1) u_{r F}+u_{r E}$ on $\left[p_{1}, p_{2}\right]$, which is easily seen to imply $u_{r E}\left(p_{2}\right)-u_{\ell F}\left(p_{2}\right)=(N-3)\left[u_{\ell F}\left(p_{2}\right)-u_{r F}\left(p_{2}\right)\right]$. If $N=3$, we define a continuous function $u_{3}\left(\cdot \mid p_{\ell}, p_{r}\right)$ on $\left[p_{\ell}, p_{r}\right]$ by setting $u_{3}\left(\cdot \mid p_{\ell}, p_{r}\right)=u_{\ell F}$ on $\left[p_{\ell}, p_{2}\right]$ and $u_{3}\left(\cdot \mid p_{\ell}, p_{r}\right)=u_{r E}$ on $\left[p_{2}, p_{r}\right]$.

If $N>3$, we proceed as in the previous paragraph to determine a belief $\left.p_{3} \in\right] p_{2}, p_{r}[$, and determine a continuous function $u_{3}\left(\cdot \mid p_{\ell}, p_{r}\right)$ that coincides with $u_{\ell F}$ on $\left[p_{\ell}, p_{2}\right]$, solves the experimenter ODE on $\left.] p_{2}, p_{3}\right]$, and coincides with $u_{r F}$ on $\left[p_{3}, p_{r}\right]$. Performing as many steps as necessary, we end up with beliefs $p_{0}=p_{\ell}<p_{1}<p_{2}<\cdots<p_{N-1}<$ $p_{N}=p_{r}$ and continuous functions $u_{1}\left(\cdot \mid p_{\ell}, p_{r}\right), \ldots, u_{N}\left(\cdot \mid p_{\ell}, p_{r}\right)$ on $\left[p_{\ell}, p_{r}\right]$ such that $u_{n}\left(\cdot \mid p_{\ell}, p_{r}\right)$ coincides with $u_{\ell F}$ on $\left[p_{\ell}, p_{n-1}\right]$, solves the experimenter ODE on $\left.] p_{n-1}, p_{n}\right]$, and coincides with $u_{r F}$ on $\left[p_{n}, p_{r}\right]$. By construction, the average of these $N$ functions coincides with $\bar{u}$.

Now consider a finite family of contiguous intervals $\left.] p_{\ell, i}, p_{r, i}\right]$ whose union equals ]$\left.p_{N}^{b}, p_{N}^{\sharp}\right]$. For each of these intervals, let $p_{n, i}$ denote the corresponding belief $p_{n}$ as determined in the previous paragraph. Define functions $u_{1}, \ldots, u_{N}$ on the unit interval by setting $u_{n}=s$ on $\left[0, p_{N}^{b}\right], u_{n}=u_{n}\left(\cdot \mid p_{\ell, i}, p_{r, i}\right)$ on $\left.] p_{\ell, i}, p_{r, i}\right]$, and $u_{n}=\bar{u}$ on $\left.] p_{N}^{\sharp}, 1\right]$. For 
$n=1, \ldots, N$, define a strategy $k_{n}$ as $k_{n}(p)=1$ if $p$ lies in $\left.p_{N}^{\ddagger}, 1\right]$ or one of the intervals $\left.] p_{n-1, i}, p_{n, i}\right] ; k_{n}(p)=0$ if $p$ lies in $\left[0, p_{N}^{b}\right]$ or one of the intervals $\left.] p_{\ell, i}, p_{n-1, i}\right]$ and ]$\left.p_{n, i}, p_{r, i}\right] ; k_{n}(p)=\bar{u}(p) /[(N-1) c(p)]$ everywhere else. Clearly, $u_{n}$ is player $n$ 's payoff function associated with the strategy profile $\left(k_{1}, \ldots, k_{N}\right)$, and $\bar{u}$ is the corresponding average payoff function. Note that by construction, $u_{1}$ is differentiable at $p_{N}^{\mathrm{b}}$ with $u_{1}^{\prime}\left(p_{N}^{b}\right)=0$.

Step 3: Ensuring mutually best responses. The following arguments establish that player $n$ plays a best response against $K_{\neg n}$ as implied by the strategy profile constructed in Step 2. First, the graph of $u_{n}$ is above $\mathcal{D}_{N-1}$ on ] $p_{N}^{\ddagger}, 1$ ], so playing $R$ is optimal against $K_{\neg n}(p)=N-1$ there. Second, $b\left(p, u_{n}\right)=c(p)$ on $] p_{N}^{\sharp}, p_{N}^{\ddagger}$ ], making $k_{n}(p)=\bar{u}(p) /[(N-1) c(p)]$ trivially optimal on this interval. Third, after increasing the number and reducing the size of the intervals ] $\left.p_{\ell, i}, p_{r, i}\right]$ if necessary, the graph of $u_{n}$ is above level $s$ and below $\mathcal{D}_{1}$ on $] p_{N}^{\mathrm{b}}, p_{N}^{\sharp}$ [, so it is optimal for player $n$ to play $R$ whenever all other players play $S$, and to play $S$ whenever one other player plays $R$. Fourth, we have $b\left(p_{N}^{\mathrm{b}}, u_{n}\right)=\lambda\left(p_{N}^{\mathrm{b}}\right)\left[u_{n}\left(j\left(p_{N}^{\mathrm{b}}\right)\right)-s\right] / r=\lambda\left(p_{N}^{\mathrm{b}}\right)\left[\bar{u}\left(j\left(p_{N}^{\mathrm{b}}\right)\right)-s\right] / r=c\left(p_{N}^{\mathrm{b}}\right)$ as the lefthand derivative of $u_{n}$ at $p_{N}^{\mathrm{b}}$ is zero, so playing $S$ is optimal at this belief. Fifth, $u_{n}(j(p))$ is at least weakly increasing and $c(p)$ is decreasing on $\left[0, p_{N}^{b}\left[\right.\right.$; therefore, $b\left(p, u_{n}\right)<c(p)$ on this interval, again implying the optimality of $S$.

Proof of Corollary 3. The proof follows the same lines as for Corollary 1 . Here we consider the case where exactly 1 of the $N$ players is playing $R$ and where a success results in the players playing symmetrically as in that corollary. The relevant ODE is

$$
\Delta \lambda p(1-p) u^{\prime}(p)+[r+\lambda(p)] u(p)=k r \lambda(p) h+(1-k) r s+\lambda(p) w(j(p))
$$

with $k=0$ for a free-rider and $k=1$ for an experimenter, and where $w$ is the function $u^{(\iota)}$ derived in Corollary 1.

We obtain equations for $u_{n}$ of the form

$$
\begin{aligned}
u_{n}(p)=d_{1}^{(\iota+1)}\left(k_{n}\right) p+ & d_{0}^{(\iota+1)}\left(k_{n}\right)(1-p)+m^{(\iota+1)}(1-p) \Omega(p)^{\mu_{N}} \\
& +\frac{1}{r} \sum_{\eta=0}^{\iota-1} l^{(\iota-\eta)} \lambda_{0}\left(\lambda_{0} / \lambda_{1}\right)^{\lambda_{0} / \Delta \lambda} \\
& \times\left(\sum_{\gamma=0}^{\eta}\left(\frac{\Delta \lambda}{r}\right)^{\eta-\gamma} \frac{\eta !}{\gamma !}\left(\ln \left[\left(\lambda_{0} / \lambda_{1}\right)^{\gamma} \Omega(p)\right]\right)^{\gamma}\right)(1-p) \Omega(p)^{\lambda_{0} / \Delta \lambda} \\
& +C_{n}^{(\iota+1)}(1-p) \Omega(p)^{\left(r+\lambda_{0}\right) / \Delta \lambda},
\end{aligned}
$$

where

$$
\begin{aligned}
d_{1}^{(\iota+1)}(k) & =\frac{\lambda_{1}}{r+\lambda_{1}}\left[\lambda_{1} h+\frac{r}{\lambda_{1}}\left(\lambda_{1} h-s\right) \iota\right]+\frac{r}{r+\lambda_{1}}\left[k \lambda_{1} h+(1-k) s\right] \\
d_{0}^{(\iota+1)}(k) & =\frac{\lambda_{0}}{r+\lambda_{0}}\left[\lambda_{0} h-\frac{r}{\lambda_{0}}\left(s-\lambda_{0} h\right) \iota\right]+\frac{r}{r+\lambda_{0}}\left[k \lambda_{0} h+(1-k) s\right] \\
m^{(\iota+1)} & =C^{(0)}\left(\frac{\lambda_{0}\left(\lambda_{0} / \lambda_{1}\right)^{\mu}}{\lambda_{0}-\mu \Delta \lambda}\right)^{\iota} \frac{\lambda_{0}\left(\lambda_{0} / \lambda_{1}\right)^{\mu}}{r+\lambda_{0}-\mu \Delta \lambda}
\end{aligned}
$$


and

$$
l^{(\iota-\eta)}=\frac{C^{(\iota-\eta)}}{\eta !}\left(-\frac{\lambda_{0}\left(\lambda_{0} / \lambda_{1}\right)^{\lambda_{0} / \Delta \lambda}}{\Delta \lambda}\right)^{\eta} \text { for } \eta=0, \ldots, \iota-1 .
$$

This leads to the representation stated in the corollary.

Proof of Proposition 7. The proof proceeds in four steps, two of which are simpler versions of the corresponding steps in the proof of Proposition 5. Let $\hat{p}_{2,1}$ denote the belief where the graph of $V_{2}^{*}$ cuts $\mathcal{D}_{1}$, and consider $I=\left[\hat{p}_{2,1}-\epsilon, p^{m}\right]$ with $\epsilon>0$ small enough that for all $\hat{p} \in I$, the unique solution to (1) with $N=2$ that crosses $\mathcal{D}_{1}$ at the belief $\hat{p}$ has positive slope there.

Step 1: Construction of the average payoff function. Fix a belief $\hat{p} \in I$. On [ $\hat{p}, 1]$, we define $u_{\hat{p}}$ as the unique solution to (1) with $N=2$ that starts on $\mathcal{D}_{1}$ at $\hat{p}$. On [ $p_{2}^{*}, \hat{p}$ [, we define $u_{\hat{p}}$ as the unique solution to the ODE

$$
\Delta \lambda p(1-p) u^{\prime}(p)+[r+\lambda(p)] u(p)=\frac{r}{2}[s+\lambda(p) h]+\lambda(p) u_{\hat{p}}(j(p))
$$

that ends on $\mathcal{D}_{1}$ at $\hat{p}$. By construction, $u_{\hat{p}}$ is continuous, $u_{\hat{p}}(p)=s+2 b\left(p, u_{\hat{p}}\right)-c(p)$ on ] $\hat{p}, 1]$, and $u_{\hat{p}}(p)=s+b\left(p, u_{\hat{p}}\right)-\frac{1}{2} c(p)$ on $\left.] p_{2}^{*}, \hat{p}\right]$.

Proceeding as in the proof of Proposition 5, we establish the existence of a $\hat{p} \in$ ] $\hat{p}_{2,1}, p^{m}$ [ such that the corresponding function $u_{\hat{p}}$ has the property that there is a belief $\bar{p} \in] p_{2}^{*}, \hat{p}\left[\operatorname{such}\right.$ that $u_{\hat{p}}(\bar{p})=s, u_{\hat{p}}(p)>s$ for $p>\bar{p}$, and $\lambda(\bar{p})\left[u_{\hat{p}}(j(\bar{p}))-s\right] / r=c(\bar{p})$.

We define a function $\bar{u}$ on $[0,1]$ by taking $\bar{u}$ equal to the function $u_{\hat{p}}$ just determined on $[\bar{p}, 1]$, and $\bar{u}=s$ everywhere else. We want to establish that $\bar{u}$ is increasing on $[\bar{p}, 1]$. The explicit representation (3) makes this obvious on [ $\hat{p}, 1]$. Suppose now that $\bar{u}$ is not increasing on $[\bar{p}, \hat{p}]$. Then there exist beliefs $p^{\prime}<p^{\prime \prime}$ in $\left.] \bar{p}, \hat{p}\right]$ such that $\bar{u}^{\prime}\left(p^{\prime}-\right) \geq 0$, $\bar{u}^{\prime}\left(p^{\prime \prime}-\right) \leq 0$, and $\bar{u}$ is weakly decreasing on $\left[p^{\prime}, p^{\prime \prime}\right]$. As $j\left(p^{\prime \prime}\right)>j\left(p^{\prime}\right)>p^{m}$, we have $\bar{u}\left(j\left(p^{\prime \prime}\right)\right)>\bar{u}\left(j\left(p^{\prime}\right)\right)$, hence $\bar{u}\left(j\left(p^{\prime \prime}\right)\right)-\bar{u}\left(p^{\prime \prime}\right)>\bar{u}\left(j\left(p^{\prime}\right)\right)-\bar{u}\left(p^{\prime}\right)$ and $b\left(p^{\prime \prime}, \bar{u}\right)>b\left(p^{\prime}, \bar{u}\right)$. This implies $\bar{u}\left(p^{\prime}\right)=s+b\left(p^{\prime}, \bar{u}\right)-c\left(p^{\prime}\right) / 2<s+b\left(p^{\prime \prime}, \bar{u}\right)-c\left(p^{\prime \prime}\right) / 2=\bar{u}\left(p^{\prime \prime}\right)$-a contradiction.

Step 2: Construction of the players' payoff functions and strategies. We define

$$
\bar{b}(p, u)=\left[\lambda(p)(\bar{u}(j(p))-u(p))-\Delta \lambda p(1-p) u^{\prime}(p)\right] / r
$$

for any left-differentiable real-valued function $u$ on $] 0,1]$.

For any two beliefs $p_{\ell}<p_{r}$ in $[\bar{p}, \hat{p}]$, Step 2 in the proof of Proposition 5 yields a belief $\left.p_{s} \in\right] p_{\ell}, p_{r}$ [ (denoted by $p_{1}$ there) as well as continuous functions $u_{1}\left(\cdot \mid p_{\ell}, p_{r}\right)$ and $u_{2}\left(\cdot \mid p_{\ell}, p_{r}\right)$ on $\left[p_{\ell}, p_{r}\right]$. Both functions coincide with $\bar{u}$ at the beliefs $p_{\ell}$ and $p_{r}$; $u_{1}\left(\cdot \mid p_{\ell}, p_{r}\right)$ solves $u(p)=s+\bar{b}(p, u)-c(p)$ on $\left.] p_{\ell}, p_{s}\right]$ and $u(p)=s+\bar{b}(p, u)$ on $\left.] p_{s}, p_{r}\right]$; $u_{2}\left(\cdot \mid p_{\ell}, p_{r}\right)$ solves $u(p)=s+\bar{b}(p, u)$ on $\left.] p_{\ell}, p_{s}\right]$ and $u(p)=s+\bar{b}(p, u)-c(p)$ on $\left.] p_{s}, p_{r}\right]$; the average of these two functions coincides with $\bar{u}$ on all of $\left[p_{\ell}, p_{r}\right]$.

It is easily seen that $u_{1}\left(\cdot \mid p_{\ell}, p_{r}\right)<\bar{u}<u_{2}\left(\cdot \mid p_{\ell}, p_{r}\right)$ on $] p_{\ell}, p_{r}[$. Moreover, using similar arguments as for the average payoff function, it is straightforward to show that $u_{1}\left(\cdot \mid p_{\ell}, p_{r}\right)$ is increasing on $\left[p_{\ell}, p_{r}\right]$ and $u_{2}\left(\cdot \mid p_{\ell}, p_{r}\right)$ is increasing on $\left[p_{\ell}, p_{s}\right]$. However, these arguments do not preclude the possibility that the function $u_{2}$ is decreasing on some interval $\left[p_{s}, p_{s}+\epsilon\right]$. 
Now consider a finite family of contiguous intervals $\left.] p_{\ell, i}, p_{r, i}\right]$ whose union equals ] $\bar{p}, \hat{p}]$. For each of these intervals, let $p_{s, i}$ denote the corresponding belief $p_{s}$ as determined above. Define functions $u_{1}$ and $u_{2}$ on the unit interval by setting $u_{n}=s$ on $[0, \bar{p}]$, $u_{n}=u_{n}\left(\cdot \mid p_{\ell, i}, p_{r, i}\right)$ on $\left.] p_{\ell, i}, p_{r, i}\right]$, and $u_{n}=\bar{u}$ on $\left.] \hat{p}, 1\right]$. Define a simple strategy $k_{1}$ by setting $k_{1}(p)=1$ if and only if $p$ lies in ] $\left.\hat{p}, 1\right]$ or one of the intervals $] p_{\ell, i}, p_{s, i}$, and a simple strategy $k_{2}$ by setting $k_{2}(p)=1$ if and only if $p$ lies in $\left.] \hat{p}, 1\right]$ or one of the intervals $\left.] p_{s, i}, p_{r, i}\right]$. Clearly, $u_{1}$ and $u_{2}$ are the payoff functions associated with the strategies $k_{1}$ and $k_{2}$, and $\bar{u}$ is the corresponding average payoff function. By construction, $u_{1}$ is differentiable at $\bar{p}$ with $u_{1}^{\prime}(\bar{p})=0$.

Step 3: Establishing that $u_{2}^{\prime}(\hat{p})>-\Delta \lambda h$. Unlike $u_{1}$, the function $u_{2}$ is not necessarily increasing on $[\bar{p}, \hat{p}]$, so we do not know whether its graph lies below the diagonal $\mathcal{D}_{1}$ to the left of $\hat{p}$, which will be important to establish the mutual best-response property in Step 4 below. Our next aim, therefore, is to show that $u_{2}^{\prime}(\hat{p})>-\Delta \lambda h$, implying that $u_{2}$ stays below $\mathcal{D}_{1}$ to the immediate left of $\hat{p}$.

We have $u_{2}(\hat{p})=s+\bar{b}\left(\hat{p}, u_{2}\right)-c(\hat{p})=s+c(\hat{p})$, hence $\bar{b}\left(\hat{p}, u_{2}\right)=2 c(\hat{p})$ and $\Delta \lambda \hat{p} \times$ $(1-\hat{p}) u_{2}^{\prime}(\hat{p})=\lambda(\hat{p})[\bar{u}(j(\hat{p}))-s-c(\hat{p})]-2 r c(\hat{p})$. As $\bar{u}(p)=\lambda(p) h+2 c(\hat{p})(1-p) /$ $(1-\hat{p})(\Omega(p) / \Omega(\hat{p}))^{\mu_{2}}$ on $[\hat{p}, 1]$, equation (2) for $N=2$ implies $\lambda(\hat{p}) \bar{u}(j(\hat{p}))=\lambda_{1}^{2} h \hat{p}+$ $\lambda_{0}^{2} h(1-\hat{p})+2\left[r / 2+\lambda_{0}-\mu_{2} \Delta \lambda\right] c(\hat{p})$. Straightforward computations now reveal that

$$
u_{2}^{\prime}(\hat{p})=\Delta \lambda h-2\left(\mu_{2}+\frac{r}{2 \Delta \lambda}+\hat{p}\right) \frac{c(\hat{p})}{\hat{p}(1-\hat{p})}
$$

and that $u_{2}^{\prime}(\hat{p})>-\Delta \lambda h$ if and only if $\hat{p}$ is larger than

$$
p^{+}=\frac{\left(s-\lambda_{0} h\right)\left(\mu_{2}+\frac{r}{2 \Delta \lambda}\right)}{\lambda_{1} h-s+\Delta \lambda h\left(\mu_{2}+\frac{r}{2 \Delta \lambda}\right)},
$$

which is easily seen to lie between $p_{2}^{*}$ and $p^{m}$. As $\hat{p}>\hat{p}_{2,1}$ (the belief where the graph of $V_{2}^{*}$ cuts $\left.\mathcal{D}_{1}\right)$, a sufficient condition for $u_{2}^{\prime}(\hat{p})>-\Delta \lambda h$ is that $\hat{p}_{2,1} \geq p^{+}$or, equivalently, $V_{2}^{*}\left(p^{+}\right) \leq s+c\left(p^{+}\right)$. Further computations show that the latter is the case if and only if

$$
\frac{\mu_{2}+\frac{r}{2 \Delta \lambda}+1}{\mu_{2}+1}\left(\frac{\mu_{2}}{\mu_{2}+1} \frac{\mu_{2}+\frac{r}{2 \Delta \lambda}+1}{\mu_{2}+\frac{r}{2 \Delta \lambda}}\right)^{\mu_{2}} \leq 2 .
$$

Now, since $r /(2 \Delta \lambda)<\mu_{2}$ and the function $h(x)=\left(\mu_{2}+x+1\right)^{\mu_{2}+1}\left(\mu_{2}+x\right)^{-\mu_{2}}$ is increasing for $x \geq 0$, the left-hand side of this inequality is bounded above by

$$
\frac{2 \mu_{2}+1}{\mu_{2}+1}\left(\frac{\mu_{2}}{\mu_{2}+1} \frac{2 \mu_{2}+1}{2 \mu_{2}}\right)^{\mu_{2}}
$$

which is clearly smaller than 2 .

Step 4: Ensuring mutually best responses. As $u_{1}$ is increasing on $[\bar{p}, 1]$, player 1 is easily seen to play a best response against $k_{2}$, irrespective of the choice of intervals [ $\left.p_{\ell, i}, p_{r, i}\right]$. First, $u_{1}$ is above $\mathcal{D}_{1}$ on $\left.] \hat{p}, 1\right]$. Second, it is above $s$ and below $\mathcal{D}_{1}$ on $] \bar{p}, \hat{p}[$. Third, we have $b\left(\bar{p}, u_{1}\right)=\lambda(\bar{p})\left[u_{1}(j(\bar{p}))-s\right] / r=\lambda(\bar{p})[\bar{u}(j(\bar{p}))-s] / r=c(\bar{p})$ as the lefthand derivative of $u_{1}$ at $\bar{p}$ is zero. Fourth, $u_{1}(j(p))$ is at least weakly increasing and $c(p)$ is decreasing on $\left[0, \bar{p}\left[\right.\right.$; therefore, $b\left(p, u_{1}\right)<c(p)$ on this interval. 
Turning to player 2 , the fact that $u_{2}^{\prime}(\hat{p})>-\Delta \lambda h$ allows us to choose a finite family of intervals $\left[p_{\ell, i}, p_{r, i}\right]$ for any $\delta>0$ such that the graph of $u_{2}$ is below the diagonal $\mathcal{D}_{1}$ on $] \bar{p}, \hat{p}$ [ and the vertical distance $u_{2}-u_{1}$ is at most $\delta$ at any belief in this interval (and hence on $[0,1])$. If we take $\delta$ sufficiently small, player 2 is now also seen to play a best response. On $[\bar{p}, 1]$, the arguments are exactly the same as for player 1 . On $\left[j^{-1}(\hat{p}), \bar{p}[\right.$, $u_{2}(j(p))=\bar{u}(j(p))$ is increasing and $c(p)$ is decreasing, hence $b\left(p, u_{2}\right)<c(p)$. On $\left[0, j^{-1}(\hat{p})\left[\right.\right.$, finally, $u_{2}(j(p)) \leq u_{1}(j(p))+\delta$ and $b\left(p, u_{1}\right) \leq b\left(j^{-1}(\hat{p}), u_{1}\right)<c\left(j^{-1}(\hat{p})\right)$, hence $b\left(p, u_{2}\right) \leq b\left(p, u_{1}\right)+\lambda(p) \delta / r<c\left(j^{-1}(\hat{p})\right)<c(p)$ for $\delta$ sufficiently small.

Proof of Corollary 4. The proof parallels that of Corollary 1 . Here we consider the general case where $K$ of the $N$ players are playing $R$, for which the relevant ODE is

$$
\Delta \lambda p(1-p) u^{\prime}(p)+\left[\frac{r}{K}+\lambda(p)\right] u(p)=k \frac{r}{K} \lambda(p) h+(1-k) \frac{r}{K} s+\lambda(p) u^{(0)}(j(p))
$$

with $k=0$ for a free-rider and $k=1$ for an experimenter. As in the proof of Corollary 1 , we take $u^{(0)}(p)=\lambda(p) h+C^{(0)}(1-p) \Omega(p)^{\mu_{N}}$ with $C^{(0)}$ being the constant that fixes payoffs above the diagonal where everyone plays $R$. Having noted that under condition (7) the recursion ends after just one iteration, i.e., with $u^{(1)}$, we obtain equations for $u_{n}$ of the form

$$
\begin{aligned}
u_{n}(p)= & \left(\lambda_{1} h \frac{\lambda_{1}}{r K^{-1}+\lambda_{1}}+\frac{r K^{-1}}{r K^{-1}+\lambda_{1}}\left[k_{n}(p) \lambda_{1} h+\left(1-k_{n}(p)\right) s\right]\right) p \\
& +\left(\lambda_{0} h \frac{\lambda_{0}}{r K^{-1}+\lambda_{0}}+\frac{r K^{-1}}{r K^{-1}+\lambda_{0}}\left[k_{n}(p) \lambda_{0} h+\left(1-k_{n}(p)\right) s\right]\right)(1-p) \\
& +C^{(0)}\left(\frac{\lambda_{0}\left(\lambda_{0} / \lambda_{1}\right)^{\mu_{N}}}{r K^{-1}+\lambda_{0}-\mu_{N} \Delta \lambda}\right)(1-p) \Omega(p)^{\mu_{N}}+C_{n}^{(1)}(1-p) \Omega(p)^{\left(r K^{-1}+\lambda_{0}\right) / \Delta \lambda}
\end{aligned}
$$

and setting $K=1, N=2$ leads to the representations stated in the corollary.

\section{REFERENCES}

Bellman, Richard and Kenneth L. Cooke (1963), Differential-Difference Equations. Academic Press, New York. [283]

Bergemann, Dirk and Juuso Välimäki (2008), "Bandit problems.” In The New Palgrave Dictionary of Economics (Steven N. Durlauf and Larry E. Blume, eds.), Palgrave Macmillan, Basingstoke. [279]

Besanko, David and Jianjun Wu (2008), "The impact of market structure and learning on the tradeoff between research competition and cooperation." Unpublished paper, School of Management, Northwestern University. [280]

Bolton, Patrick and Christopher Harris (1999), "Strategic experimentation." Econometrica, 67, 349-374. [276, 279, 283, 284, 285, 288, 299]

Bolton, Patrick and Christopher Harris (2000), "Strategic experimentation: The undiscounted case." In Incentives, Organization, and Public Economics (Peter J. Hammond and Gareth D. Myles, eds.), 53-68, Oxford University Press, Oxford. [277, 279] 
Cohen, Asaf and Eilon Solan (2009), "Bandit problems with Lévy payoff processes.” Unpublished paper, Department of Statistics and Operations Research, Tel Aviv University. Available at http://arxiv.org/abs/0906.0835v1. [283]

Davis, Mark H. A. (1993), Markov Models and Optimization. Chapman \& Hall, London. [280]

Décamps, Jean-Paul and Thomas Mariotti (2004), "Investment timing and learning externalities." Journal of Economic Theory, 118, 80-102. [280]

Hopenhayn, Hugo and Francesco Squintani (2008), "Preemption games with private information.” Unpublished paper, Department of Economics, University of Essex. [280]

Keller, Godfrey, Sven Rady, and Martin Cripps (2005), "Strategic experimentation with exponential bandits.” Econometrica, 73, 39-68. [276, 277, 278, 279, 280, 282, 283, 284, $285,294]$

Klein, Nicolas (2010), “Strategic learning in teams.” Unpublished paper, Munich Graduate School of Economics. [279]

Klein, Nicolas and Sven Rady (2008), "Negatively correlated bandits.” Unpublished paper, University of Munich. [279]

Malueg, David A. and Shunichi O. Tsutsui (1997), "Dynamic R\&D competition with learning.” RAND Journal of Economics, 28, 751-772. [279]

Moscarini, Giuseppe and Francesco Squintani (2007), "Competitive experimentation with private information: The survivor's curse.” Unpublished paper, University of Essex. [280]

Presman, Ernst L. (1990), "Poisson version of the two-armed bandit problem with discounting." Theory of Probability and Its Applications, 35, 307-317. [280]

Presman, Ernst L. and Isaac M. Sonin (1990), Sequential Control With Incomplete Information: The Bayesian Approach to Multi-Armed Bandit Problems. Academic Press, London. [280]

Rothschild, Michael (1974), "A two-armed bandit theory of market pricing." Journal of Economic Theory, 9, 185-202. [279]

Strulovici, Bruno (2010), "Learning while voting: Determinants of collective experimentation.” Econometrica, 78, 933-971. [279]

Submitted 2009-7-23. Final version accepted 2010-1-13. Available online 2010-1-13. 